Utah State University

DigitalCommons@USU

$6-24-2013$

\title{
Comprehensive Theoretical Framework for Modeling Diverse Electron Transport Experiments in Parallel Plate Geometries
}

\author{
Alec Sim \\ Irving Valley College \\ JR Dennison \\ Utah State Univesity
}

Follow this and additional works at: https://digitalcommons.usu.edu/mp_conf

Part of the Physics Commons

\section{Recommended Citation}

Alec M. Sim and JR Dennison, "Comprehensive Theoretical Framework for Modeling Diverse Electron Transport Experiments in Parallel Plate Geometries," Paper Number, AIAA-2013-2827, 5th AIAA Atmospheric and Space Environments Conference, San Diego, CA, June 24-27, 2013, 31 pp. DOI: 10.2514/ 6.2013-2827

This Conference Paper is brought to you for free and open access by the Materials Physics at DigitalCommons@USU. It has been accepted for inclusion in Conference Proceedings by an authorized administrator of DigitalCommons@USU. For more information, please contact digitalcommons@usu.edu.

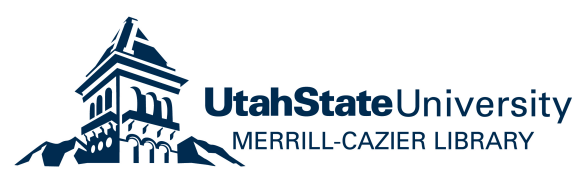




\title{
Comprehensive Theoretical Framework for Modeling Diverse Electron Transport Experiments in Parallel Plate Geometries
}

\author{
Alec M. Sim ${ }^{1}$ \\ Irvine Valley College, Irvine, CA, 92604 \\ J.R. Dennison ${ }^{2}$ \\ Utah State University, Logan, UT, 84322
}

A unified set of parameters and dynamic equations have been developed to describe the time-dependent surface voltage and currents measured for a broad range of electron transport experiments conducted in parallel plate geometry with a dielectric slab above a grounded electrode and with either a floating or fixed voltage upper surface. The framework can model measurements of constant voltage, time-of-flight and AC conductivity; radiation induced conductivity; surface voltage accumulation and decay; electrostatic discharge; electron emission and electron-induced luminescence. The broad applications of the theoretical framework are outlined in terms a comprehensive classification of the ways in which charge is injected into or excited within a material; these classifications include surface deposition, bulk deposition and penetrating radiation for pulsed, stepped and periodic applied voltages/charge from either surface electrodes or electron beams. A set of equations are developed to model evolving electron transport and related phenomena in highly disordered insulating materials over large ranges of time, electric field, temperature, absorbed dose, and adsorbed dose rate. These analytic equations derived from physics-based theories predict the equilibrium and time-dependent accumulation, dissipation and transport of charge carriers; these basic equations are (i) Gauss' law, (ii) a 1D electron continuity equation with Ohm's law and source terms, (iii) a $1 D$ continuity equation for holes with source terms, and (iv) the sum of currents due to various conduction mechanisms (including contributions from drift, diffusion, dispersion, polarization, and radiation-induced processes). The total conductivity is modeled as the sum of contributions from three independent conductivity mechanisms: thermally activated hopping, variable range hopping, and radiation-induced conductivity using a concise, unified set of independent fitting parameters. At a microscopic level, modeling and understanding these conduction mechanisms in disordered insulating materials is fundamentally based on a detailed knowledge of the distribution and occupation of the density of states (DOS) of nearly-free and trapped charged carriers. The conduction is controlled by transitions between extended valence and conduction band states, between localized trap states and the extended valence and conduction band states, and hopping between localized states; constant, linear, power law, exponential and Gaussian localized DOS are considered. By analyzing the observed temperature, field, dose rate and time dependent conductivities that result from both extended and localized trap state conduction, this theoretical framework provides new insight into the role of the localized trap state DOS in myriad ground-based materials testing methods.

\section{Nomenclature}

$a$

$a_{B}$

$\mathrm{A}$

A beam

$\mathrm{B}$

$b_{r}$

$b_{t}$

c

d

$\mathrm{D}$

$\dot{D}$

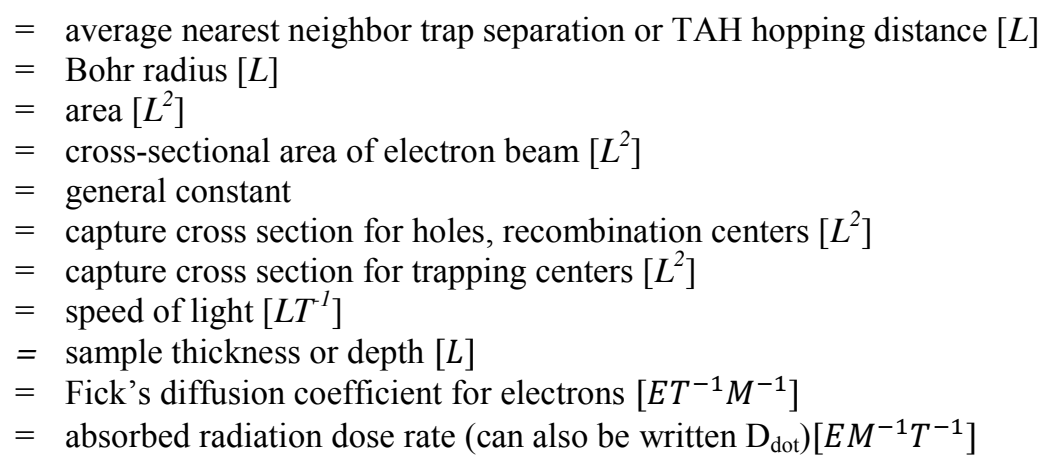

\footnotetext{
${ }^{1}$ Assistant Professor, Physics Department, 5500 Irvine Center Drive, Irvine CA, 92618, Member.

${ }^{2}$ Professor, Physics Department, UMC 4415, Senior Member.
} 


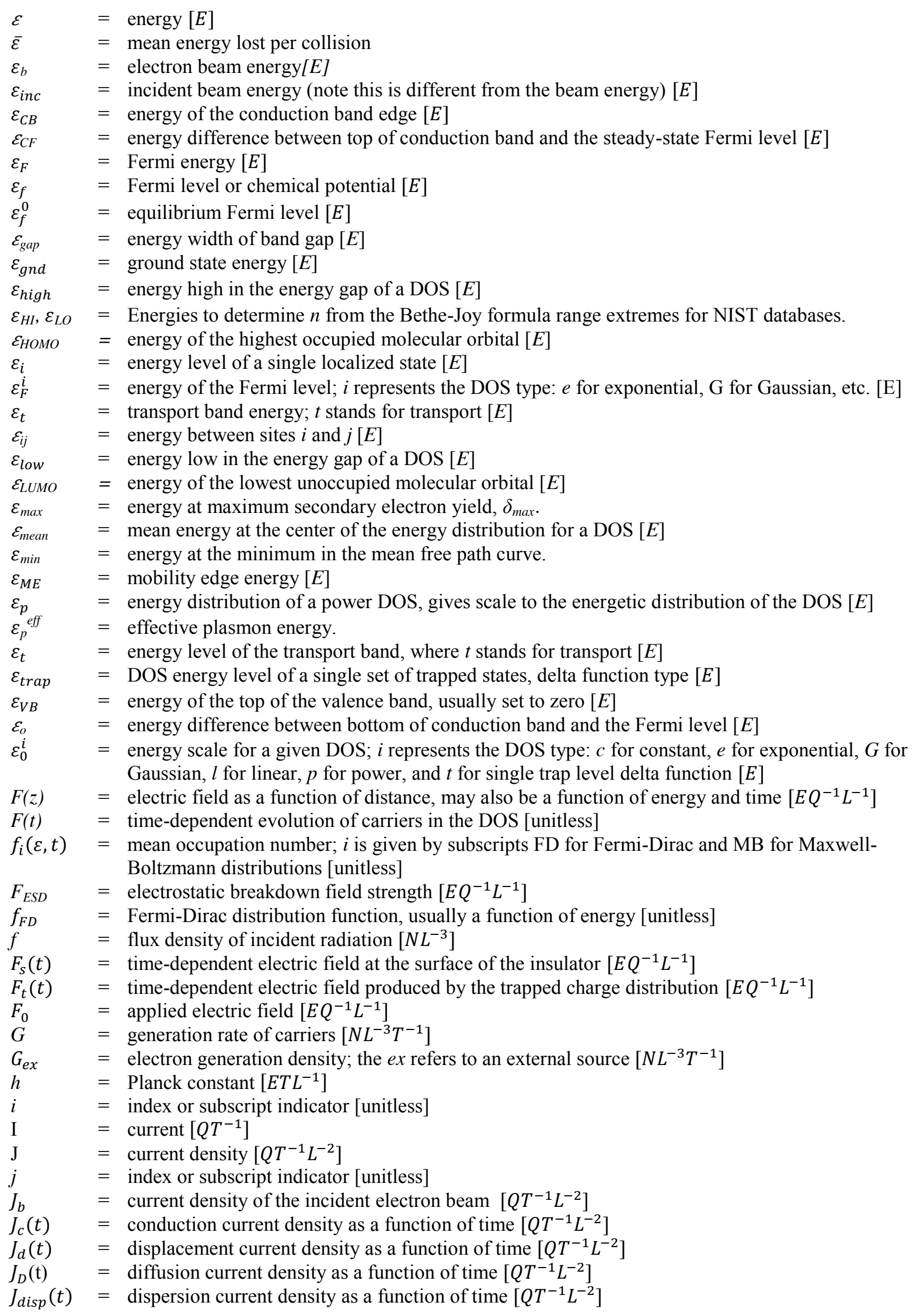


$J_{\text {inj }}(t)=$ injected current density as a function of time $\left[Q T^{-1} L^{-2}\right]$

$J_{\text {Leak }}(t)=$ leakage current density as a function of time $\left[Q T^{-1} L^{-2}\right]$

$J_{\text {meas }}(t)=$ current density as a function of time measured at the rear electrode $\left[Q T^{-1} L^{-2}\right]$

$J_{p}(t)=$ polarization current density as a function of time $\left[Q T^{-1} L^{-2}\right]$

$J_{p 0} \quad=$ maximum (initial) polarization current density as a function of time $\left[Q T^{-1} L^{-2}\right]$

$J_{p}^{\downarrow}(t) \quad=$ decrease in polarization current density as a function of time $\left[Q T^{-1} L^{-2}\right]$

$J_{p}^{\uparrow}(t) \quad=$ increase in polarization current density as a function of time $\left[Q T^{-1} L^{-2}\right]$

$J_{s}(t) \quad=$ surface injection current density at the surface that enters the sample as a function of time $\left[Q T^{-1} L^{-2}\right]$

$J_{\text {sat }} \quad=$ equilibrium saturation current density that exits the sample at the rear electrode $\left[Q T^{-1} L^{-2}\right]$

$J_{s d} \quad=$ steady state current density as measured at the rear electrode, not to be confused with surface injection current density or time-dependent current density $\left[Q T^{-1} L^{-2}\right]$

$J_{\text {tot }}(\mathrm{t})=$ total current density as a function of time $\left[Q T^{-1} L^{-2}\right]$

$J_{0} \quad=$ initial current density $\left[Q T^{-1} L^{-2}\right]$

$K=$ Empirical constant in the Bethe Joy formula.

$\mathrm{K}\left(z_{p}\right)=$ number of electrons deposited per unit time per unit volume, where $z_{p}$ is the penetration depth $\left[N E^{-1} L^{-3}\right]$

$k_{b} \quad=$ Boltzmann constant $\left[E K^{-1}\right]$

$M_{A} \quad=$ Atomic weight.

$m_{e} \quad=$ Electron rest mass.

$N=$ Stopping power exponent.

$N_{A} \quad=$ Avogadro's number.

$N(\varepsilon) \quad=$ density of localized states per unit energy per unit volume $\left[N E^{-1} L^{-3}\right]$

$N_{A} \quad=$ number of participating particles at site $\mathrm{A}$ [unitless]

$N_{B} \quad=$ number of participating particles at site B [unitless]

$n_{e} \quad=$ density of free carriers per unit volume $\left[N L^{-3}\right]$

$n_{e}(\varepsilon, t)=$ density of free carriers per unit energy per unit volume $\left[N L^{-3}\right]$

$N_{e}=$ total density of electron conduction states within $k_{b} T$ of the CB per unit volume $\left[N L^{-3}\right]$

$N_{i n j}=$ total density of injected carriers $\left[N L^{-3}\right]$

$n_{h}(t)=$ density of immobile holes per unit volume $\left[N L^{-3}\right]$

$n_{t} \quad=$ density of trapped carriers per unit volume, not the total $\left[N L^{-3}\right]$

$n_{t}(\varepsilon, t)=$ density of trapped carriers (occupied trap states) per unit energy per unit volume $\left[N E^{-1} L^{-3}\right]$

$N_{t} \quad=$ total density of (available) localized states per unit volume; (not a trapped carrier), $N_{t}=\int N(\varepsilon) d \varepsilon\left[N L^{-3}\right]$

$n_{t o t}(z, t)=$ total density of carriers per unit volume as a function of spatial position and time $\left[N L^{-3}\right]$

$N_{v} \quad=$ total density of valence states per unit volume $\left[N L^{-3}\right]$

$\mathrm{P} \quad=$ a constant, usually a power [unitless]

$\mathrm{P} \quad=$ polarization density $\left[Q L^{-4} E M^{-1} T^{2}\right]$

$q_{i} \quad=$ charge per carrier, where $i$ is the type of carrier: $\mathrm{e}=$ electron, $\mathrm{h}=$ hole, ex $=$ exiton, etc. $[Q]$

$\mathrm{Q}_{i n j} \quad=$ injected charge per unit area $\left[Q L^{-2}\right]$

$\mathrm{Q}_{\text {acc }} \quad=$ accumulated charge $[Q]$

$\mathrm{r}=$ radius to some point $\mathrm{P}$ in the sample or in space $[L]$

$R=$ range of a carrier; this can apply to the range of an incident particle penetrating into a material or the range of an electron as it hops from one localized state to the next, typically a mean value $[L]$

$S(r, t)=$ volume charge rate density creation term; has dimensions of $\left[L^{-3} T^{-1}\right]$

$s_{c} \quad=$ capture cross section of conduction electrons by fixed holes $\left[L^{2}\right]$

$S_{C S D A}=$ Electron stopping power in CSDA

$\mathrm{t} \quad=$ time $[T]$

$T \quad=$ temperature $[K]$

$t_{\tau} \quad=\quad$ transit time, time for carriers to cross the sample thickness $d[T]$

$T_{0} \quad=$ characteristic dispersion of states in a given DOS; always coupled with $k_{b}$ to give energy $[K]$

$T_{0}^{i}=$ characteristic dispersion of states in a given DOS; i represent DOS type: e for exponential, $l$ for linear, $\mathrm{G}$ for Gaussian $[K]$

$\mathrm{V} \quad=$ volume $\left[L^{-3}\right]$

$V \quad=$ electric potential $\left[E Q^{-1}\right]$

American Institute of Aeronautics and Astronautics 


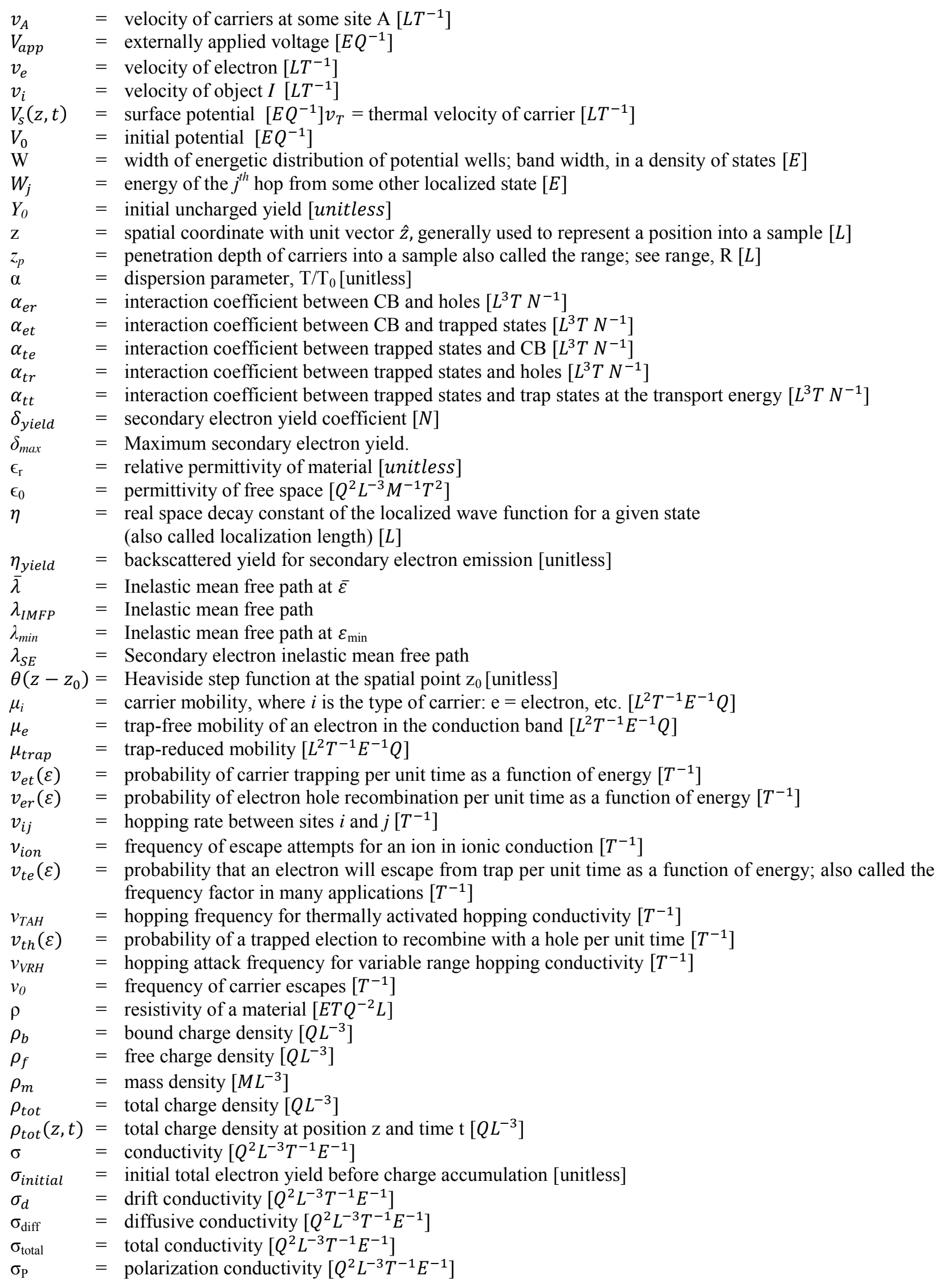




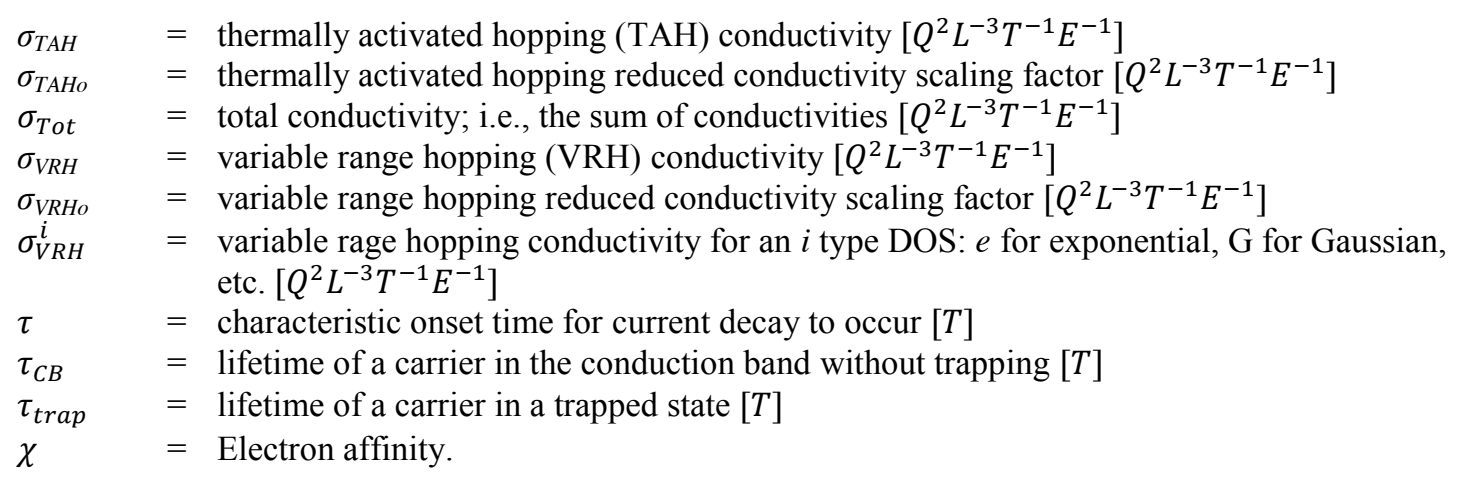

\section{Introduction}

Spacecraft in orbit are exposed to intense plasma environments and high energy particles. Charging to high

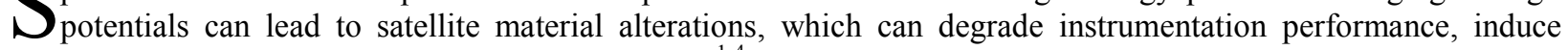
systems failures, and create potential safety hazards. ${ }^{1-4}$ The ubiquity of highly insulating materials in the design of spacecraft and many other technology components places special emphasis on understanding and modeling the electrical properties of the insulators. Detailed study of experimental data and physical models are critical for predicting and mitigating potentially damaging charging phenomena. ${ }^{5-6}$ Developing a better understanding of the physics of insulating materials, increasing the versatility and reliability of charge transport models, and expanding the database of information for the electronic properties of insulating materials provides assistance for spacecraft designers in accommodation and mitigation of these harmful effects. ${ }^{2,7}$ In this paper we explore the following relevant concepts: simplified parallel plate charging geometry, unification of theoretical charge transport models in a single formalism using a common nomenclature and physically based parameters set, density of states (DOS) driven charge transport, and charge injection models. Using these concepts, myriad charge transport and spacecraft charging effects can be modeled.

The complex relationships between spacecraft insulators and their surroundings are fundamentally based on a detailed knowledge of how individual materials store and transport charge. The key to mitigating these effects is an understanding of the time required to dissipate harmful charge imbalances on and within the material used in spacecraft construction. ${ }^{8}$ For simple systems considered here the charge decay time is a function of the material conductivity, $\sigma(F, \dot{D}, T, t)$, which in turn is a function of electric field $F$, incident flux $f$, time $t$, dose rate $\dot{D}$, and material temperature $T$ : that is, $\tau_{\text {decay }} \propto \sigma(F, \dot{D}, T, t)^{-1}$.

The conductivity of a material is the key transport parameter in determining how deposited charge will redistribute throughout the system, how rapidly charge imbalances will dissipate, and what equilibrium potential will be established under given environmental conditions. ${ }^{9}$ Further, the conductivity connects the physical make up of a material with the number of available carriers, their type, and how mobile charge is within the material. It is the low charge mobility of insulators that causes charge to accumulate where deposited, preventing uniform redistribution of charge and creating differential local potentials. Through careful experimental applications we can develop an understanding of the relationship between material make up, carrier type, carrier density and the effects on carrier mobility.

The Utah State University (USU) Material Physics Group (MPG) has been developed to specifically address the spacecraft community's concerns for the charging of materials. ${ }^{10-14}$ The USU MPG has built an extensive knowledgebase of the behaviors observed in many spacecraft materials. ${ }^{15}$ This database ${ }^{16}$ in addition to application of theoretical models, has been implemented in engineering tools used in spacecraft design. ${ }^{15}$ The accumulation of nearly two decades of work has provided the USU-MPG with a unique platform from which to study the spacecraft charging problem at the material level. Each of the experimental systems has been designed to test specific material behavior or phenomena that result from composite behavior. Fig. 1 is a graphical representation of the experimental systems and their dependence on conductivity $\sigma$, temperature $T$, electric field $F$, time $t$, frequency $\omega$, and dose rate $\dot{D}$. In each of these applications, the USU-MPG has implemented theoretical models to describe the observed behavior.

Many engineering models currently in use for spacecraft design are largely static in their predictions and therefore new models based on the dynamic physics-largely developed for photoconductors, high voltage power applications, and electrets-must be applied to make significant improvements in predicting time-dependent behaviors. Consider a spacecraft near the danger point, which undergoes a high energy event—say a sudden high 


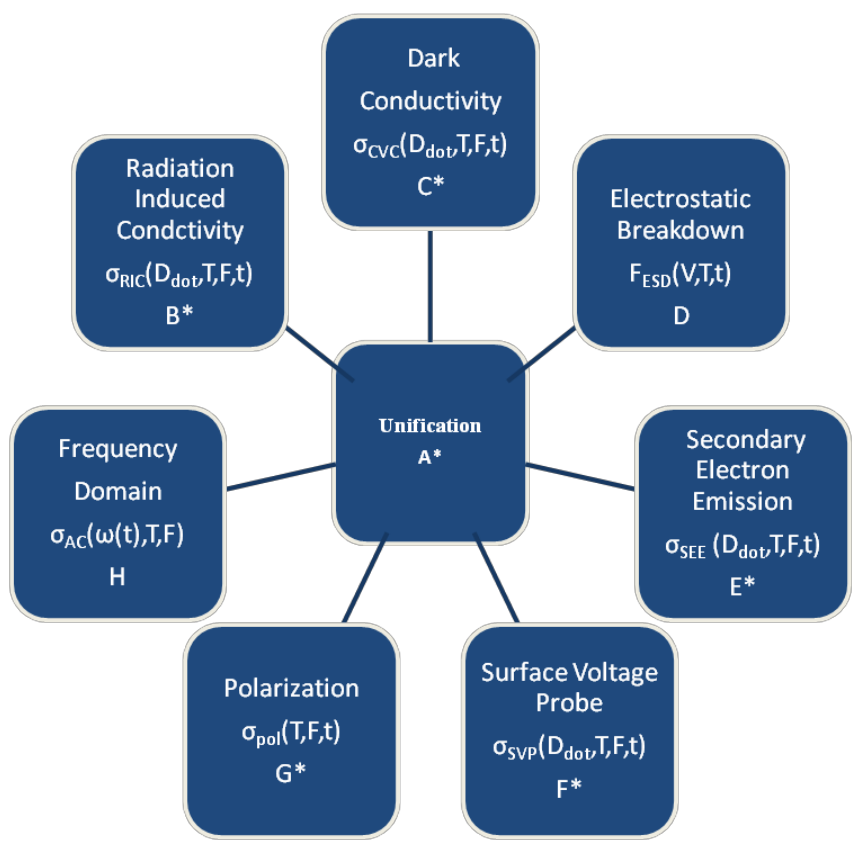

Figure I. Materials Physics Group experimental organization diagram and conduction mechanisms. Each box represents a specific conduction mechanism associated with a specific set of experimentally measured parameters. (A) Model unification (Sim, 2013). (B) RIC = Radiation Induced Conductivity (Guthrie, 2013). (C) DCC = Dark Current Conductivity (Brunson, 2008; Dekany, 2009). (D) ESD = Electrostatic Breakdown (C Sim, 2010). (E) SEE = Secondary Electron Emission (Hoffmann, 2009). (F) SVP = Small Voltage Probe (Hodges, 2012). (G) POL=Impulse polarization studies (Brunson, 2009). (H) FD = Frequency domain polarization studies.

flux of energetic particles. Depending on the charge deposition rate, induced dissipation rate, and net local field the event may cause a system failure. In addition, the large majority of models do not include the effects of low temperature behavior of materials, where the mobility may be reduced by several orders of magnitude due to structural phase transitions or changes in the physical transport mechanisms. For many highly insulating materials there is a transition in the mobility where the conduction process is no longer driven by pure thermal processes involving the conduction band, but rather is a process that proceeds purely by localized quantum mechanical interactions. Most materials are not well characterized for low temperatures, intermediate dose-rates, and very high fields. The need for a dynamic description of spacecraft charging for all time scales, temperature ranges, injection types, and field dependencies is evident.

The USU MPG has developed a consistent theoretical formalism that describes material behavior across many experimental systems. In this publication an overview of the key elements and advances of the USU MPG theoretical models are presented. This is not intended to be a complete description of the theory; for a more complete description, the interested reader should consult Refs. 17-25.

We first present a discussion of idealized experimental configurations. This facilitates the introduction of the theory from an experimental point of view. The theoretical development begins with simple conduction processes, idealized band structure, microscopic carrier transport, and macroscopic transport. It uses this physical foundation to develop a compartmentalized method for understanding the interaction of charge transport and finally the transport equations. The transport equations are extended to include all of the experimental configuration needed to study single layer thin film spacecraft materials, which are typically highly insulating disordered materials (HDIM).

\section{Experimental Configurations}

The large majority of experimental systems involved in testing HDIM can be categorized as shown in Fig. 2. Two injection modes are identified, surface electrode injection (red boxes) and charge beam injection (blue boxes). Surface electrode injection typically uses a simple parallel plate capacitor configuration that injects charge into the material via an electrode(s) and an applied field leading to charge injection effects, electron transport phenomena, 


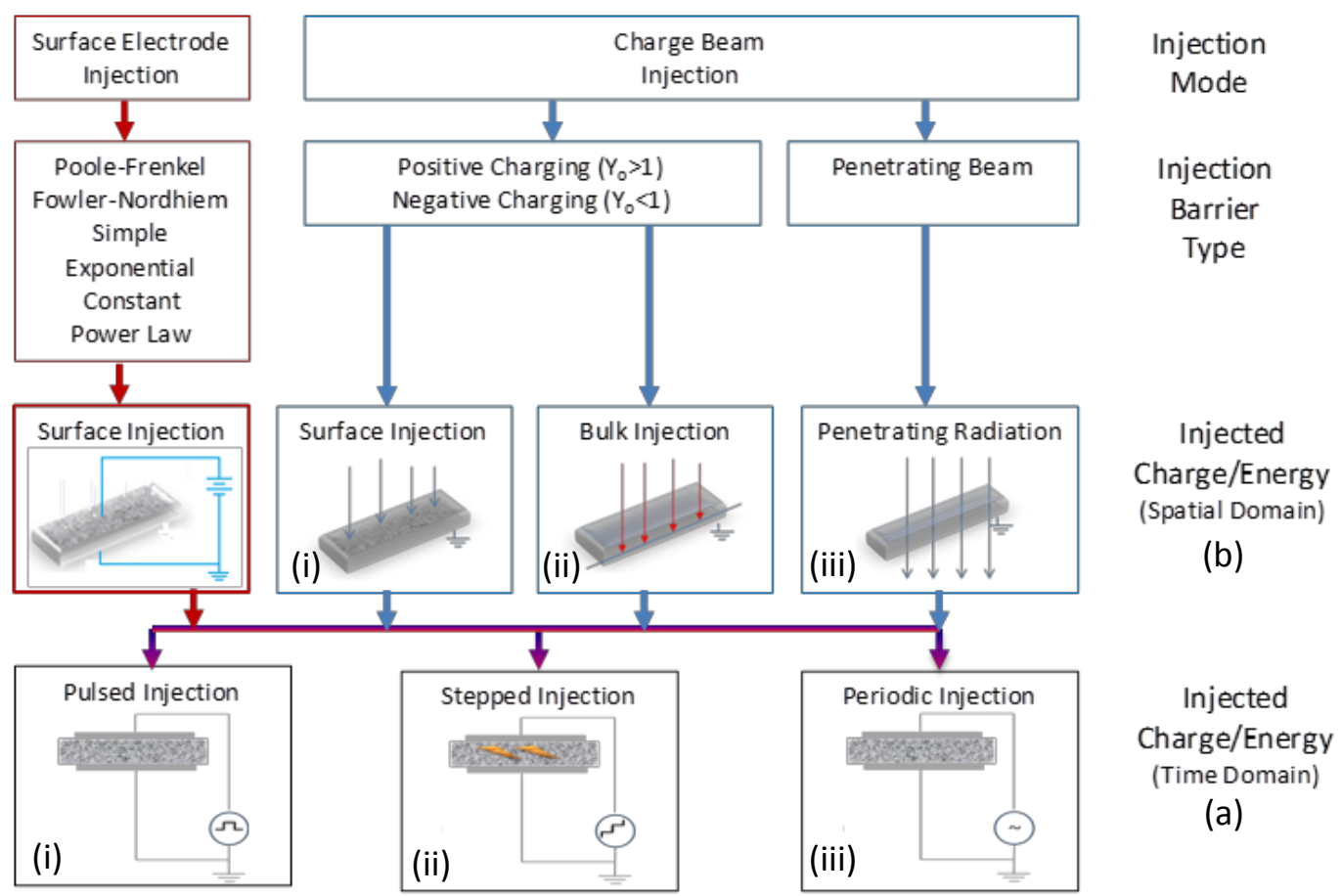

Figure 2. Simple characterization of experimental configurations. These diagrams provide a comprehensive classification of experimental configurations that describe all the common ways in which charge and energy can be injected into a material. Electrode injection modes are outlined in red. Charge beam injection modes are outlined in blue. Four types of charge/energy injection in the spatial domain are identified. Hence, there are twelve combinations of chargelenergy injection experimental configurations in this classification scheme.

polarization, and electrostatic breakdown. This type of experiment is very general and the interested reader can find the details of the USU experimental system in Ref. 18. In the charge beam injection experimental configuration, a charged particle (electron or ion) beam or a photon source is incident on the material. Depending on the specifics of the deposition process, charge and/or energy is deposited or removed from the sample and different physical phenomena are observed. ${ }^{18,8}$ Charge beam injection is a more complex process. It includes the possibility of induced charge emission from the incident surface and the possibility the incident charge is deposited on the surface, deposited within the material, or penetrates fully through the material depositing only energy. Together, four different types of charge and energy injection are identified in Fig. 2 for the spatial domain of deposited charge. Each of these spatial modes can combine with three types of charge and energy injection in the time domain (black boxes): pulsed injection, stepped injection and periodic injection. This combination delineates 12 different experimental configurations for the "simple" parallel plate capacitor configuration that are all described by the theoretical framework outlined in this paper. Specifics of the periodic injection configurations will be discussed in subsequent publications.

Consider a charge source - such as an electron beam —incident on a highly insulating spacecraft material sample. Three experimental configurations (blue boxes) are depicted in Fig. 2: (i) low incident beam energies, where charge is deposited or released from the surface and surface charging/discharging, time of flight (TOF), surface drift and diffusion, and low energy secondary electron emission (SEE) can be studied; ${ }^{19-21}$ (ii) intermediate energies, where charge is deposited within the material and SEE, SCLC, dispersion, diffusion, drift, charge trapping due to bulk states, and electrostatic discharge (ESD), plus photoemission such as luminescence are studied; and (iii) higher energies, where incident charge completely penetrates the material depositing only energy and leading to radiation induced conductivity (RIC), dispersion, recombination, bulk state trapping, electroluminescence, and ESD are studied.

Given the six configurations indentified in Fig. 2 and the simple parallel plate geometry framework for the fields and currents in Fig. 3, a systematic theoretical development is achieved that allows for a physical understanding of the observed phenomena, characterization of material parameters across a wide array of experimental platforms, 
prediction of behavior for spacecraft materials under a wide variety of conditions, and for the first time provides an avenue by which multiple physical phenomena and their effects on spacecraft materials can be predicted and results of measurements compared for the different methods. In what follows we present an outline of the theoretical basis used to understand charge transport in these configurations and the equations developed for the system current and voltage observables. To understand how the current density equation can be studied across experimental systems all the currents that can occur in any arrangement of systems must be considered. Having considered all the current densities and resulting potentials, a macroscopic model that includes the complex disordered nature of the material is needed.

\section{Introductory theory}

How can we model charge transport and the resulting conductivity in highly disordered insulators as a function of material interactions with incident radiation dose $\dot{D}$, applied electric field $F$, temperature $T$, position of deposited charge $z$, and time $t$ ? In practice the problem is very complex and requires a detailed understanding of the microscopic mechanism that lead to macroscopic-measurable-behavior. Using the simplification that the system can be treated as a parallel plate capacitor yields a consistent pathway by which a complete theoretical formalism can be applied across many experimental system. In order to accomplish this, models for the internal and external currents, internal and external potentials, deposited charge as a function of depth in the sample $\mathrm{z}$, charge ejected from the sample surface, internal and external fields, polarization, disordered nature of the material, and any of the six configurations presented in Fig. 2 must be brought together.

\section{A. Conduction processes}

We can write the generic observed current density as $J=\sigma F$, where $\sigma$ is the conductivity. The conductivity of a single carrier is defined as $\sigma=q n \mu$, where $q$ is the charge of the carrier, $n$ is the number of carriers, and $\mu$ is the mobility. Since the conductivity contains all the information about the material response, to first order, Ohm's law defines a simple average relationship that connects the applied field to changes in the material behavior. The observed current however is due to many processes, linear and non-linear. There are a large number of mechanisms that can contribute to the observed current: Ohmic drift, polarization, spacecharge limited current (SCLC), hopping processes, diffusion, dispersion, radiation induced conductivity (RIC), secondary electron emission, (SEE) etc. We can write total current density as the sum of individual current densities as

$$
J_{\text {total }}=J_{\text {ohmic }}+J_{\text {diffusion }}+J_{\text {dispersion }}+\sum_{i}^{n} J_{i}
$$

Each of these $i$ processes can be categorized by considering whether they are the result of other fundamental processes or are fundamental. If the process is one that involves no other process, hopping as an example, then it is fundamental in nature. Processes that involve more than one fundamental process, secondary electron emission (SEE) as an example, are multi-component. In HDIM the processes of drift, diffusion, dispersion, and trapping are all dependent on carrier scattering, hopping or hopping-like interactions within the DOS. Radiation induced conductivity (RIC) and luminescence are generally multi-step processes that involve transitions from the conduction or shallow-trap-states to deep-states or the valence band. Processes like (SEE) and electrostatic breakdown; (ESD) are composites that involve deep trapping or distortion of the DOS due to high charge density or high field effects ${ }^{29-}$ 33

To understand how the current density equation can be studied across experimental systems all the currents that can occur in any arrangement of systems must be considered. Figure 3 is a representation of all the currents densities that can be expected for an experimental configuration. For example, a simple parallel plate configuration in which the sample is placed between two electrodes and an external voltage is applied is found by setting $V_{\text {ext }}=$ $V_{b}=V_{d}=0$, this is consistent with configuration (b) of Fig. 2. If the experimental configuration is that of a SEE chamber then all the current densities are needed, consistent with configuration (a) of Fig. 2. Note that in both configuration (a) and (b) there is a penetration depth $\mathrm{R}$ for charge. Having considered all the current densities and resulting potentials a macroscopic model that includes the complex disordered nature of the material is needed.

\section{B. Band structure and disorder}

The most familiar picture of an insulator and its band structure is show in Fig 4 (a). In this picture many atoms are brought together and their bonding and anti-bonding orbital's interact causing discrete electron energy states of the individual atoms to form quasi-continuous bands separated by well-defined band gaps. The band gap is the energy range where no extended electron states exist (forbidden region) and in a perfect insulator no electrons or 


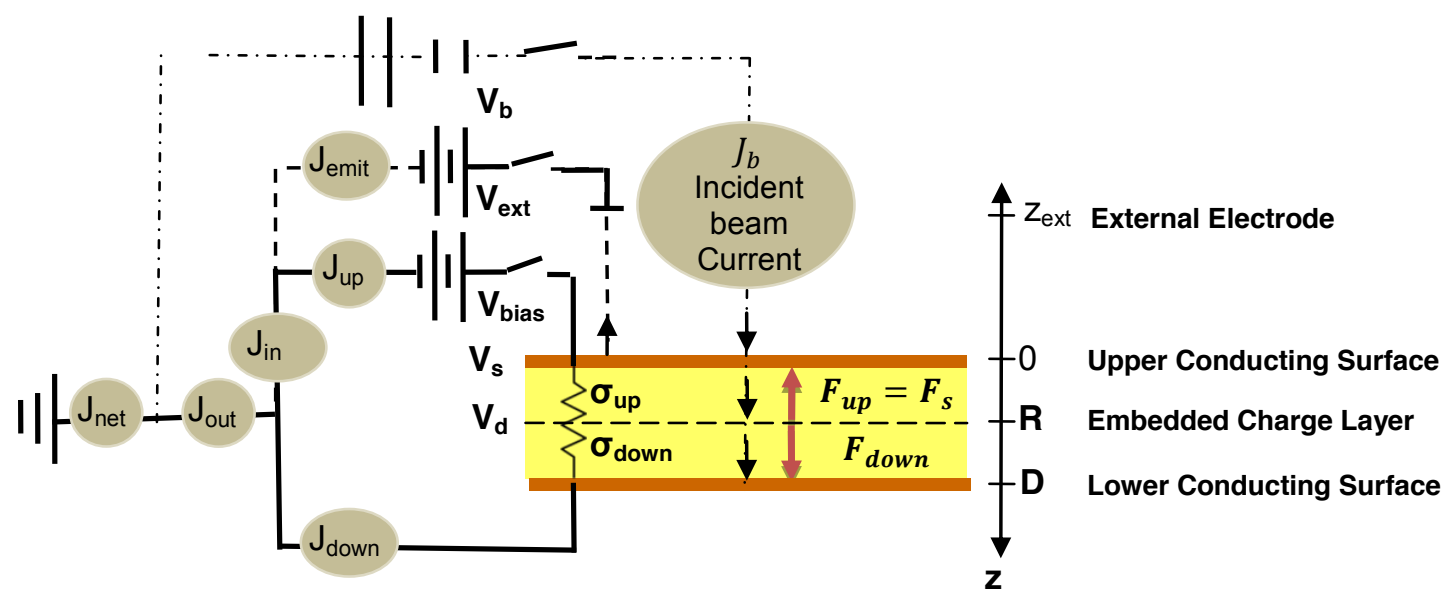

Figure 3. Basic slab geometry of charge transport for an incident electron beam or parallel plate capacitor . Electron beam can be incident on a dielectric slab with a grounded lower surface and grounded or floating upper surface. $R$-range or penetration depth; $D$-sample thickness; $F_{u p}$ and $F_{\text {down }}$-electric fields above and below embedded charge layer; J-current densities including injected beam current density, $J_{\text {in }}$, emitted current density, $J_{\text {emit }}$, current density through from upper surface, $J_{\text {up }}$, current density through lower grounded surface, $J_{\text {down }}$, injection current density, $J_{\text {inj }}$, and net total current density out of the dielectric, $J_{\text {out }}=J_{\text {emit }}+J_{\text {up }}+J_{\text {down }}$; $V$-voltages including deposited layer voltage, $V_{d}$, surface voltage, $V_{s}$, bias voltage, $V_{\text {bias }}$, external electrode voltage, $V_{\text {ext }}$, and electron beam voltage, $V_{b}$.

carriers can exist. However, variations in the periodic order in crystals and to larger extent polymers, either spatial or energetic, cause extended states to become localized in space. The disorder results from concentrations of impurity atoms, the geometry of polymer chains, their impurities, and the general random nature of their polymeric structure. Further, the polymer chains do not lend themselves to the simplifications of a lattice construct and have a myriad of structural and internal degrees of freedom. Additionally, polar groups attached to the chains, cross linking and broken bonds have significant influence on carrier mobility ${ }^{26,27}$. In most cases these localized states occur in the forbidden gap and hence form trap states. Localized states within the band gap are separated from the extended states by a mobility edge, which for HDIM is an effective conduction band edge. This high level of disorder leads to a density of states (DOS), with complex energetic and positional dependencies within the band gap. The effects of disorder are depicted in Fig 4 (b) along with the mobility edge. Often in the literature the conduction band edge and valance band edge are confused with the mobility edge. The mobility edge is often at a different energy that is generally closer to the center of the band gap; notice also that the term band gap is no longer appropriate and one should really refer to this region as the localized state region. In order to provide a salient discussion for the connection between HDIM and charge transport a simplified model is used to describe the bang gap and transport states shown in Fig 4 (c). The DOS or trapping states are represented as a series of filled or empty circles and the level to which the band gap states are filled is given by $\varepsilon(F, T, t, \dot{D})$, referred to as the Fermi-level. A complete discussion of trap filling (thermalization) and response of the Fermi-level will not be presented here the interested reader should consult Ref 22.

Figure 5 shows an idealization of the effects of disorder on the DOS. Here $N(\varepsilon)$ is the DOS as a function of energy and $\mu(\varepsilon)$ is the mobility as a function of energy. The mobility is determined by wave function overlap. Thus, when $N(\varepsilon)$ is such that wave function interaction is small the states in the gap become localized ${ }^{28}$. The DOS states are categorized into three regions, conduction, shallow, and deep. In conduction states the carriers move freely as $N(\varepsilon)$ and $\mu(\varepsilon)$ are high. In the second region $N(\varepsilon)$ is still fairly high but $\mu(\varepsilon)$ begins to drop due to localization onset, thus this point is defined as the mobility edge. In the third region both $N(\varepsilon)$ and $\mu(\varepsilon)$ are low and states in the gap are localized. The measured current density often determined by interactions between conduction states and carriers trapped in the DOS. For example, carriers in trapped in localized states can escape by thermal excitation to the conduction band, (CB). Carriers in the mid to deep localized states only escape via four mechanisms; (a) thermally activated hopping $(\mathrm{TAH})^{15}$, (b) variable range hopping $(\mathrm{VRH})^{15,16}$, (c) radiation induced conductivity $(\mathrm{RIC})^{26}$, (d) recombination $^{22}$. Without radiation and for high temperature, we expect that the (TAH) mechanism will dominate as excitation from shallow traps near the mobility edge is possible. For much lower temperatures only (VRH) is possible, thus carriers proceed by hopping, (tunneling) between states whose position and energy dependent wave 
Groups of Atoms

(a)

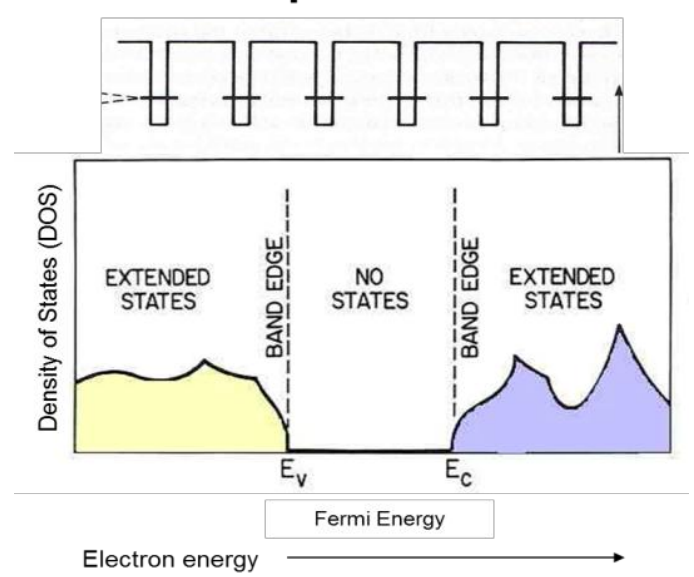

Transition to a Disordered System (b)

Simplified model (c)
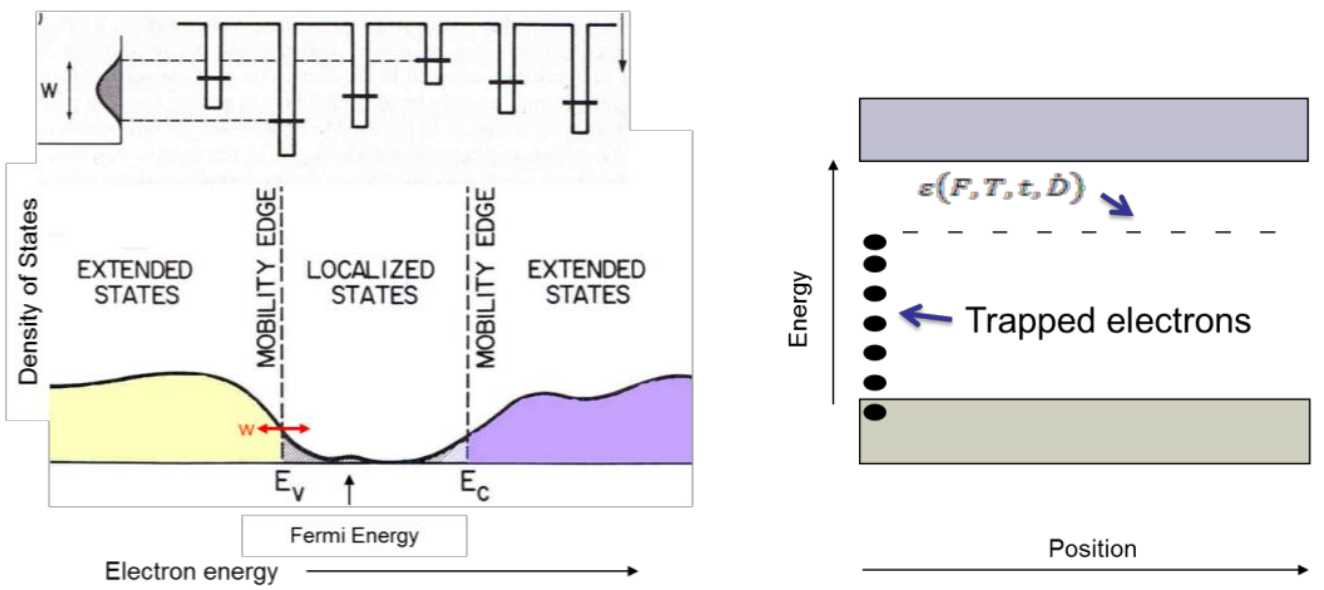

Figure 4. Idealized disordered density of states and band gap model. (a) Periodic Solids: As larger numbers of atoms are brought together their bonding and anti-bonding orbital's interact causing discrete electron energy states of the individual atoms to form quasi-continuous bands separated by well defined 'band gaps'. The band gap is an energy range in a solid where no electron states exist. (b) Disordered Solids: Variation in the periodic order in a crystal, either spatial or energetic, causes extended states to become localized in space. In most cases these localized States will move into the forbidden band gap and hence form trap states. Localized states within the band gap are separated from extended states by a mobility edge.(c) Simplified model: the extended states are depicted as simple regions and the DOS as a simple set of empty or filled states.

functions have an appropriate amount of overlap. The range of the hop will change as a function of the energy and shape of the DOS, thus different regions of the DOS can act in different ways producing different behaviors. It is very important to point out that for very low temperatures (such as those spacecraft experience) VRH is a dominant mode of charge transport. It turns out that VRH can be modeled in a similar manor to TAH using similar transport equations. VRH and subsequent charge transport covers a large class of behaviors which will not be discussed here. The interested reader should see Ref 22 or Ref 41.

The dynamics of charge trapping, de-trapping, excitation, and recombination are governed by the type of DOS that forms in the bang gap. For example, the charge release time-scale is generally dependent of the energetic depth of potential wells formed as a result of disorder. Figure 5 depicts a DOS with a changing shape. The key idea here is that the DOS can be modeled with functions of energy. The shape of the DOS affects the rate at which charge will be trapped or released, undergo recombination, and controls luminescent phenomena. Table 1 contains the most 


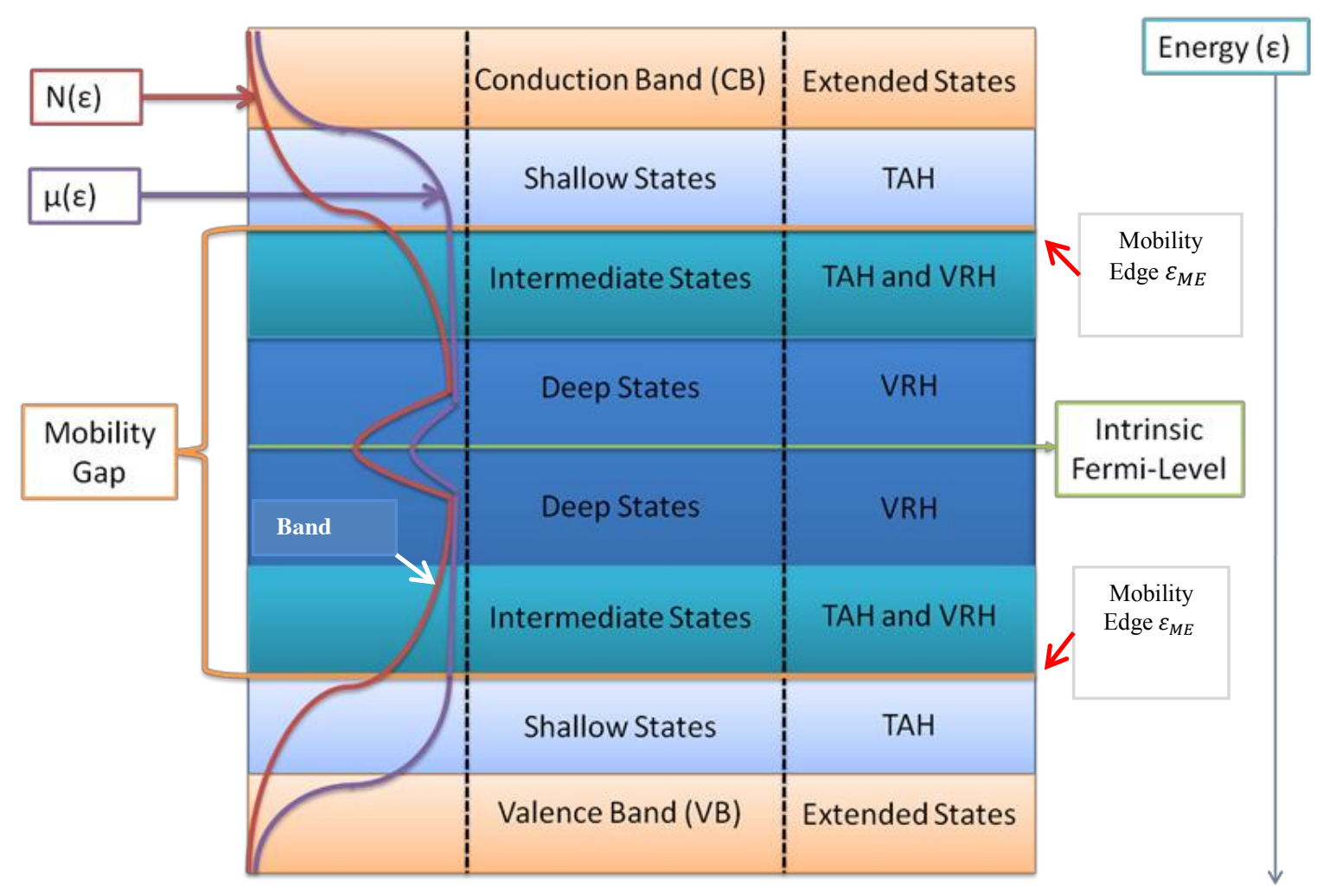

Figure 5. Composite density of states and mobility plot contrasted and mechanisms. Figure identifies the regions within the DOS that participate in specific temperature-dependent phenomena; this is contrasted with the resulting mobility. Thermally activated hopping TAH controls the high-temperature behavior and variable range hopping (VRH) controls the low-temperature behavior. Typical band conduction begins at the mobility edge and increases with increasing energy; below the mobility edge (in the mobility gap) conduction proceeds by hopping.

common types of DOS used in calculating interactions and resulting behaviors. Each DOS model is characterized by an energy scaling or width factor, $\varepsilon_{0}^{i}$ (where $i$ is the type of DOS), and $\varepsilon_{0}^{t}$ (the first moment, based on the centroid of the distribution, given by $\frac{\int_{0}^{\infty} \varepsilon N(\varepsilon) d \varepsilon}{\int_{0}^{\infty} N(\varepsilon) d \varepsilon}$. For example, $\varepsilon_{0}^{p}$ is the scaling energy for the power law DOS.

The interested reader may be concerned that the use of un-normalized DOS models will lead to erroneous values when performing calculations, which is correct, and unfortunately has occurred many times in the literature as many authors are concerned only with general behavior and not the magnitudes involved. The process of normalizing a given DOS is the same as for any such process; calculate the integral of the DOS distribution over the energetic interval that it spans, divide by the total DOS, and make sure you get one, i.e., $\int_{\varepsilon_{\text {low }}}^{\varepsilon_{\text {ligh }}}\left(\frac{N(\varepsilon)}{N_{t}}\right) d \varepsilon=1$. This normalization is specific to the type of DOS and the region of energy that it spans; therefore, it should be calculated in each specific case! The normalization is not computed here, but the DOS models are provided in Table 1 along with a few representative centroid calculations; these are NOT complete centroid calculations. The reader is referred to Ref 23 for calculations of normalization and moments of the distributions in Table 1.

Given a description of the DOS how can the macroscopic behavior that is the measured current density, resultant electric field, or potential be calculated? In order to answer this question it is clear that the microscopic behavior in the DOS must be considered to obtain macroscopic results.

\section{Microscopic Transport}

The microscopic description of charge transport, and thus the observed current or voltage is dependent on stochastic processes between individual atomic or molecular sites. These processes are driven with the energy supplied by the phonon spectrum, particle flux (where radiation is present) and electric field, $\boldsymbol{F}$. It is then the interaction of atomic or molecular wave functions, effects of the applied field, $N(\varepsilon)$, and $\mu(\varepsilon)$ that determines the 
Table 1. Types of Densities of State. The major types of DOS functions that are used in calculations for HDIM. Each DOS is given in units of number per energy per length. The scale factor determines the energy scale range of the DOS and the centroid gives the effective center of the DOS.

\begin{tabular}{|c|c|c|c|}
\hline $\begin{array}{l}\text { Type of DOS } \\
\text { Function } \\
\end{array}$ & DOS Expression & $\begin{array}{l}\text { Scale Factor } \\
\text { (Width) }\end{array}$ & $\begin{array}{c}\text { Centroid } \\
\text { (Examples Only) }\end{array}$ \\
\hline a) Delta & $N(\varepsilon)=N_{t} \delta\left(\varepsilon_{0}^{t}-\varepsilon\right)$ & $\varepsilon_{0}^{G} \rightarrow 0$ & $\varepsilon_{0}^{t} \quad$ All intervals \\
\hline b) Constant & $N(\varepsilon)=\left(N_{t} / \varepsilon_{0}^{c}\right)$ & $\varepsilon_{0}^{c}$ & $\varepsilon_{0}^{t} \sim \frac{\varepsilon_{0}^{c}}{2} \quad$ Finite interval \\
\hline c) Linear & $N(\varepsilon)=\frac{N_{t}}{\varepsilon_{0}^{l^{2}}} \varepsilon$ & $\varepsilon_{0}^{l}$ & $\begin{array}{c}\varepsilon_{0}^{t} \sim\left(\frac{2}{3}\right) \varepsilon_{0}^{l} \quad \text { Finite } \\
\text { interval }\end{array}$ \\
\hline d) Power & $N(\varepsilon)=\frac{N_{t}}{\varepsilon_{0}^{p}}\left(\varepsilon / \varepsilon_{0}^{p}\right)^{P}$ & $\varepsilon_{0}^{p}$ & $\begin{array}{c}\varepsilon_{0}^{t} \sim\left(\frac{P+1}{P+2}\right) \varepsilon_{0}^{p} \quad \text { Finite } \\
\text { interval }\end{array}$ \\
\hline e) Exponential & $\begin{array}{c}N(\varepsilon)=\left(N_{t} / \varepsilon_{0}^{e}\right) \exp \left(-\varepsilon / \varepsilon_{0}^{e}\right) \\
\varepsilon_{0}^{e} \equiv k_{b} T_{0}^{e}\end{array}$ & $\begin{array}{c}\varepsilon_{0}^{e} \\
(1 / e \text { width) }\end{array}$ & $\begin{array}{c}\varepsilon_{0}^{t} \sim \varepsilon_{0}^{e} \\
\text { inf inite interval only }\end{array}$ \\
\hline f) Gaussian & $N(\varepsilon)=\frac{N_{t}}{\varepsilon_{0}^{G} \sqrt{2 \pi}} \exp \left(-\left(-\varepsilon_{0}^{t}\right)^{2} / 2 \varepsilon_{0}^{G^{2}}\right)$ & $\varepsilon_{0}^{G}$ & $\begin{array}{c}\varepsilon_{0}^{t} \\
\text { infinite interval only }\end{array}$ \\
\hline
\end{tabular}

observed transport. Consider two atomic states $\eta$ and $\eta$ one of which is occupied by a carrier (perhaps an electron), and the other which is empty. In this case, there are two possibilities. First, the electron will escape via thermal excitation and is either recaptured or excited to the conduction states. The second occurs when phonon contribution is small compared with the wave function interaction between the sites and the charge will "hop" between states. The change in the probability for a given site $\eta$ to be occupied is given by the Pauli master equation, (PME)

$$
\frac{\partial p(\eta, t)}{\partial t}=\sum_{\dot{\eta}}\left(U_{\dot{\eta}, \eta} p(\eta, t)-U_{\eta, \dot{\eta}} p(\eta, t)\right) .
$$

where $p(\eta, t)$ is the probability that an atomic state is occupied at time $t$ and $U_{\eta, \dot{\eta}}$ is the transition probability per unit time for $\eta \stackrel{\text { trans }}{\longrightarrow} \eta$. Many authors have shown the connection between the macroscopic phenomena resulting in and the microscopic (PME) or some variation of the (PME) $)^{30-32}$.

In principle the PME can be applied to any material as it is an atomistic picture. However, it is most useful in materials with low to modest disorder where estimation of the wave function interaction is more accurate. In spacecraft materials - particularly polymeric insulators - we often encounter disorder that is too great for the PME to be useful in practice. Thus we rely on mean field approximations to estimate the results. The current USUMPG models ${ }^{29,33}$ used to describe VRH conductivity ${ }^{15}$ are based on mean field approximation techniques ${ }^{34-40}$. It has been shown that the PME does yield macroscopic transport equations similar to those found for TAH, diffusion, dispersion, and drift. A comprehensive review is given by Ref 42. The mean field theories are adequate for describing the connection between the microscopic transport and macroscopic behavior. The development of a macroscopic description of charge transport theory with an average microscopic approach is needed. To that end a review of Maxwell's equations in a relevant form is prudent.

\section{Macroscopic transport}

The connection between microscopic average behavior and macroscopic measurable values must account for all the charge entering and leaving the system. Therefore, a discussion of macroscopic transport follows from Maxwell's equations and the charge continuity equation.

$$
\nabla \cdot \boldsymbol{D}=\rho_{c}
$$




$$
\begin{gathered}
\nabla \cdot \boldsymbol{B}=0 \\
\nabla \times \boldsymbol{F}=-\frac{\partial \boldsymbol{B}}{\partial t} \\
\nabla \times \mathbf{H}=\mathbf{J}_{\boldsymbol{c}}+\frac{\partial \mathbf{D}}{\partial \mathrm{t}} \\
\nabla \cdot \mathbf{J}_{\boldsymbol{c}}+\mathrm{S}(\mathbf{r}, \mathbf{t})=-\frac{\partial \rho_{t o t}}{\partial \mathrm{t}}
\end{gathered}
$$

Here $\rho_{c}$ is the free charge density, $\rho_{\text {tot }}$ is the total charge density, $\rho_{b}$ (shown below) is the bound charge density, $\boldsymbol{D}$ is the electric displacement field, $\boldsymbol{B}$ is the magnetic field, $\boldsymbol{r}$ is the three-dimensional position vector, $S(\boldsymbol{r}, t)$ is the injected current that becomes either space charge or migrating trapped charge, and $\boldsymbol{H}$ is the magnetizing field. Only in rare cases is the effect of the magnetic field considered; thus, in general we concern ourselves only with Eq. (1) and Eq. (5). Note that inherent in these equations is the constitutive relation that relates the total charge density, displacement field and the polarization given by:

$$
\begin{gathered}
\rho_{t o t}=\rho_{b}+\rho_{c} \\
\boldsymbol{D}=\epsilon_{0} \boldsymbol{F}+\boldsymbol{P} \equiv \epsilon_{0} \epsilon_{\mathrm{r}} \boldsymbol{F} \\
\rho_{b}=-\nabla \cdot \boldsymbol{P}
\end{gathered}
$$

Here $\rho_{\text {tot }}$ stands for the total charge, $\rho_{b}$ is the bound charge due to polarization, and $\mathrm{P}$ for polarization. $\epsilon_{0}$ and $\epsilon_{\mathrm{r}}$ are the permittivity of free space and the relative permittivity, respectively. Since the current is the sum of effects produced by all species of carriers, molecular, and atomic sites, the charge density can be written as

$$
\rho_{i}=q_{i} n_{i} .
$$

Here $i$ denotes the charge species, $q_{i}$ is the electronic charge per carrier, and $n_{i}$ is the charge carrier concentration. Note that we can refer here to charge in trapped states, bands, free charge, or even surface charge. For example, consider a system where we have injected a known amount of electron charge per unit volume, $\rho_{\text {tot }}$. Further assume that some of the charge resides in the CB and some in trapped states in the band gap; then we can write the charge density as $\rho_{t o t}=\rho_{e}+\rho_{t}$ or the number density as $n_{t o t}=n_{e}+n_{t}$.

The difficulty in determining the flavor of macroscopic equations to use is a reflection of the complexity of the material and its environment, boundary conditions, experimental setup, DOS models, and population statistics of the DOS and conduction band. One simplification that is effective in reducing the complexity of working with these equations is to reduce the $3 \mathrm{D}$ expression to a $1 \mathrm{D}$ expression. In addition, an approximation for the observed current is needed to describe how the charge concentrations change with distance and time. By doing this, expressions for the resulting electric field or potential and their time dependence can be obtained.

\section{E. Trapped charge interactions}

Consider Fig. 6, in which six transition processes are depicted, both as diagrams and mathematical models. The basic picture of the band model described in Fig. 4 is retained in Fig. 6. Note that the DOS can be any of the DOS models described in Table 1. For convenience, it will be assumed that the spatial dependence of the trapped carriers is negligible (i.e., the trap distribution is homogeneous and isotropic); as the transport equations are developed, it will become clear how to include additional dependencies, such as charge position within the material. Each of the processes can be characterized by a product of the probability of occurrence, number of available states and number of carriers that can take part in the process.

The transition rate processes depicted in Fig. 6 are: (a) excitation by thermal or external sources (RIC) across the band gap, (b) trapping of carriers in extended states, (c) excitation (de-trapping) of trapped carriers, (d) recombination of carriers in extended states with immobile holes in the VB, (e) interstate transitions, and (f) recombination of carriers in deep trapped states with holes in the VB. In process (a), thermal excitation across the large band gap in HDIMs is negligible and therefore, only external sources (excitation) are considered here.

The generation of carriers - specifically electrons-in the material, $G_{e x}(z)$, due to an incident electron beam (or any external source), is a function of distance into the material. Beyond a penetration depth, $\mathrm{z}_{\mathrm{p}}$, in the material excited electrons, carriers are no longer generated; but, charge carriers are deposited from the beam. To distinguish these two processes, the number of electrons deposited per unit time is given by $K\left(z_{p}\right)^{43-50}$. These terms allow the model to specifically address the differences depicted in Fig. 2 (i), (ii), and (iii). Understanding the physics of the deposition and generation terms allows for the inclusion of SEE and RIC processes into out model.

13

American Institute of Aeronautics and Astronautics 


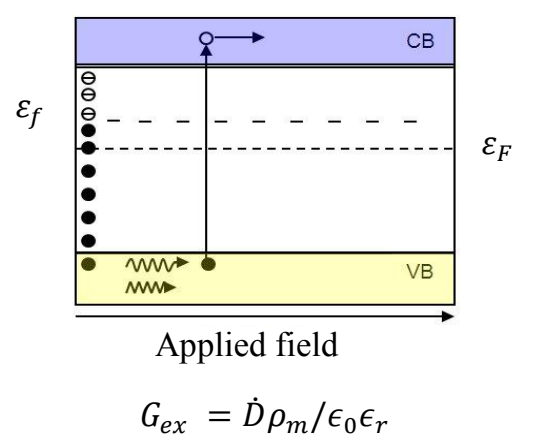

(a) Excitation by thermal or external sources

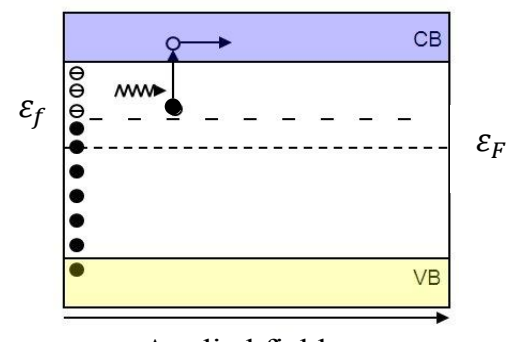

$$
\int_{\varepsilon_{F}}^{\varepsilon_{c}} \alpha_{t e}(\varepsilon) N_{c} n_{t}(\varepsilon, t) d \varepsilon f(\varepsilon)
$$$$
\text { Applied field }
$$

(c) De-trapping due to thermal excitation

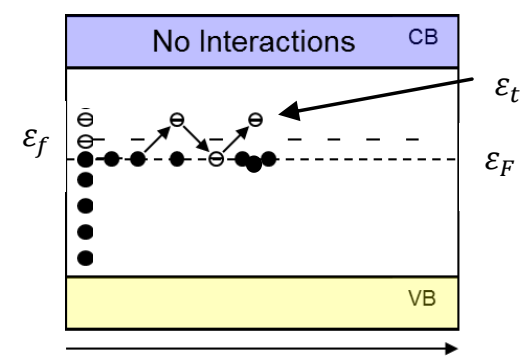

Applied field

$$
\alpha_{F T E} n_{t}\left(\varepsilon_{F}\right) N_{T E}\left(\varepsilon_{T E}\right) P_{F T E}\left(\varepsilon_{F}\right)
$$

(e) Low temperature VRH

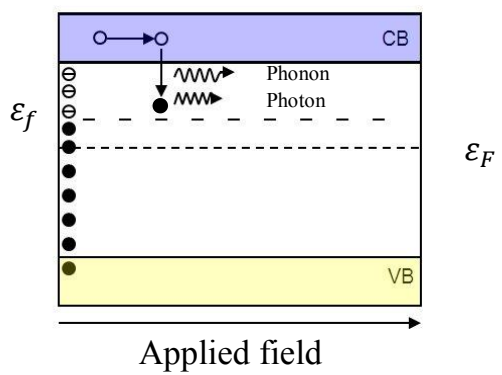

$$
\alpha_{e t}(\varepsilon) n_{e}(t)\left[N_{t}(\varepsilon)-n_{t}(\varepsilon, t)\right]
$$

(b) Trapping

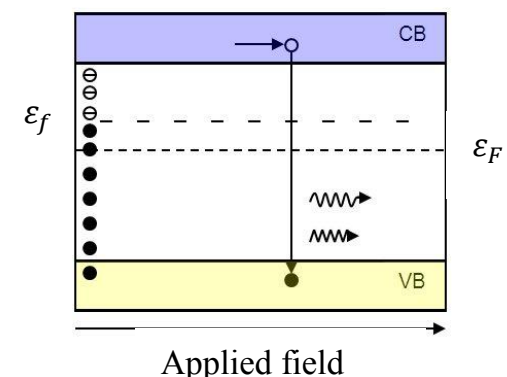

$$
\alpha_{e r}(\varepsilon) n_{e}(t)\left[n_{e}(t)+n_{t}(\varepsilon, t)\right]
$$

(d) Recombination

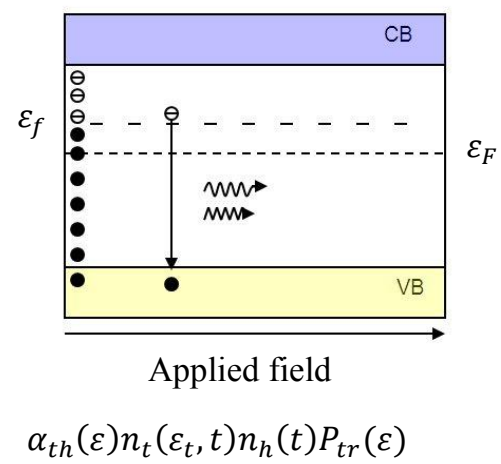

(f) Mid-band recombination

Figure 6. The six easy pieces of transport in HDIM. Free electrons are denoted by an empty circle, empty traps by a dash, and filled traps by a black circle. The vertical line of dark and open circles represent the distribution of filled and empty trap states. (a) Thermal processes or external irradiation that delivers energy sufficient to excite carriers across the band gap, creating holes in the valence band. (b) Carriers in the conduction band become trapped by localized states via thermalization (c) Carriers are re-excited into the conduction band from trapped states. (d) Carriers in the conduction band recombine with a hole in the valence band. (e) Low temperature transport; the CB is replaced by the effective transport energy (TE). (f) Low-temperature recombination that limits the flow of carriers in the transport band.

Thermal excitations (phonons) are considered only in process (c), whereas the release of phonons and photons are considered in processes (b), (d), and (f). It should be noted that any process in which an electron liberates energy is a candidate for emission of light leading to processes such as induced luminescence and arc events. A 
discussion of the process (f) and the probability of this transition occurrence, $P_{t r}(\varepsilon)$ (which is very small), and the mathematical description of (e) are left for subsequent publication, as they apply only to low-temperature conductivity and represent VRH and applications of the PME, see discussion in Section IIIC. The key piece of information to take away from processes (e) and (f) is that, they are governed by transition rates to and from trapped states.

Here a connection between microscopic transport behavior found in VRH processes and macroscopic transport as depicted in (a)-(d) is made. For VRH charge transport occurs in a narrow energy range centered around the transport energy (TE). Charges trapped in deep states hop to the transport states from the Fermi-level $\varepsilon_{F}$. Thus (e) suggests that deep states act as a trapped charge distribution and the TE is a kind of low mobility conduction band. This implies that if we develop a set of macroscopic transport equations for diagrams (a)-(d) we can apply the results to (e). Note that the material parameters for VRH, such as the mobility, are not the same as their high temperature counter parts. The process depicted in (f) occurs only for very low temperatures and is not considered here. The interested reader should consult Ref 41.

Figure 6 and the resulting discussion provide a simple way to discuss interactions between a trapped charge distribution and conduction states. Therefore, using the ideas of Section III C,D, and E a description of charge transport for HDIM can be presented.

\section{F. Transport Equations}

In this section a set of transport equations are developed using only drift and diffusion current densities and a set of equations describing trap limited transport for the RIC application. This is done to provide a specific application of the theory but is not a complete development that can be applied to any system. For extensions of these ideas and applications of extended transport equations see Ref 22. Starting with Gauss's law $\nabla \cdot \boldsymbol{D}=\rho_{c}$, the continuity equation $\nabla \cdot \mathrm{J}+\mathrm{J}_{\mathrm{inj}}=-\frac{\partial \rho_{\text {tot }}}{\partial \mathrm{t}}$, an expression for the current density with both the drift and diffusion currents, and

The processes in Fig. 6 represent all the interactions with disorder necessary for describing the transport of electrons in HDIM. Thus, the sum of processes (transition rates) involved in a given application will yield the appropriate transport equations. In addition to the changes in the relative concentration of electrons in the extended and localized states, a set of conservation equations that connect the total number of electron carriers in the material at any time, $t$, is needed:

$$
n_{t o t}(t)=n_{e}(t)+n_{t}(t)
$$

and the total number of electrons trapped in all the localized states at all possible trap depths and at some position, $z_{p}$, is given by

$$
n_{t}\left(\mathrm{t} ; z_{p}\right)=\int_{\varepsilon_{\text {low }}}^{\varepsilon_{\text {high }}} n_{t}\left(\varepsilon, t ; z_{p}\right) d \varepsilon
$$

,where $\varepsilon_{\text {low }}$ and $\varepsilon_{\text {high }}$ refer to the top and bottom of the DOS that contains the trapped electrons.

At this point we can look at a specific application to develop an expression for the transport equations. Assume that electrons are excited from the VB to the CB, leaving holes in their absence, but that no deposition occurs. Such behavior can be expected for penetrating radiation, such as in Fig. 2 (a) (iii). Developing the trapping equations is now a simple matter of summing over the processes in Fig. 6 that relate the change in the number of electrons in extended and localized states. To obtain the time rate of change in the number of electrons in the CB due to interactions with disorder (trapping and release of electrons), we integrate the energy-dependent processes in Fig 6: (b), (c), and (d), then sum (a) - (d) - (b) + (c) to obtain,

$$
\frac{d n_{e}(t)}{d t}=G_{e x}\left(t, z_{p}\right)-\alpha_{e r} n_{e}(t)\left[n_{e}(t)+n_{t}(t)\right]-\alpha_{e t} n_{e}(t)\left[N_{t}-n_{t}(t)\right]+\int_{\varepsilon_{f}^{0}}^{\varepsilon_{C B}} \alpha_{t e}(\varepsilon) f(\varepsilon) N_{e} n_{t}(\varepsilon, t) d \varepsilon
$$

Here, for the first time, it has been explicitly suggested that the generating term $G_{e x}\left(t, z_{p}\right)$ depends on time which can result from the time dependence of incident charge, surface potentials resulting from trapped charge distributions etc. The distribution function, $f(\varepsilon)$, is left in a general form and is not yet assumed to have a certain type of statistical nature, such as Fermi-Dirac or Maxwell-Boltzmann. For the time rate of change in the number of trapped electrons, $n_{t}(t)$, we again perform integration over energy and do the sum over processes (b) - (c) to obtain the charge trapping equations developed in Fig. 6 we can write down a set of one dimensional non-liner differential transport equations that describe the nature of charge transport in space craft materials. 


$$
\frac{d n_{t}(t)}{d t}=\alpha_{e t} n_{e}(t)\left[N_{t}-n_{t}(t)\right]-\int_{\varepsilon_{f}^{0}}^{\varepsilon} \alpha_{t e}(\varepsilon) f(\varepsilon) N_{e} n_{t}(\varepsilon, t) d \varepsilon .
$$

Without the explicit integration of the energy-dependent terms, the equations cannot be reduced further. The energy dependence of the interaction coefficient is not well understood in HDIM and mention of it is sparse in the literature; it is, therefore, assumed that the interaction coefficient is a constant for this discussion. The excitation from a trap state is governed by the distribution function, $f(\varepsilon)$, which, for the electron system, is most generally the Fermi-Dirac (FD) distribution. The FD distribution will be approximated by the Boltzmann distribution (MB), i.e.., $\alpha_{t e} N_{e} f_{M B}(\varepsilon)=v_{0} f_{M B}(\varepsilon) \approx v_{0} \exp \left[-\frac{\varepsilon}{k T}\right]$.

Equations (12) and (13) can be greatly simplified by assuming that $\alpha_{e r}$ is equal to $\alpha_{e t}$. These two terms deal with (i) recombination of electrons in the $\mathrm{CB}$ to the VB holes created during generation and (ii) the trapping of electrons in the DOS, respectively. Many authors have used this assumption, but with little or no justification ${ }^{22}$. It is possible, that since the electrons involved in these two processes always start in the $\mathrm{CB}$, that the coefficients are determined predominantly by the properties of the $\mathrm{CB}$, in which case the assumption is valid.

It is additionally assumed that the process (e) in Fig. 6 is only important at very low temperatures and that process (f) is negligible in the study of RIC. These assumptions are certainly not always true; however, many of the transport equations were developed for large fluence near room temperature and in semiconductor applications where (e) and (f) do not typically make large contributions to the observed macroscopic current. These assumptions have been noted here so that the reader will understand how the complete transport equations are related to the more common reduced set found in the literature. Keeping these assumptions in mind, we can write Eq. (12) and Eq. (13) as follows:

$$
\frac{d n_{e}(t)}{d t}=G_{e x}-\alpha_{e r} n_{e}(t)\left(n_{e}(t)+n_{t}(t)\right)+\int_{\varepsilon_{f}^{0}}^{\varepsilon_{C B}} v_{0} \exp \left[-\varepsilon / k_{b} T\right] n_{t}(\varepsilon, t) d \varepsilon
$$

and

$$
\frac{d n_{t}(t)}{d t}=\alpha_{e t} n_{e}(t)\left[N_{t}-n_{t}(t)\right]-\int_{\varepsilon_{f}^{0}}^{\varepsilon C B} v_{0} \exp \left[-\varepsilon / k_{b} T\right] n_{t}(\varepsilon, t) d \varepsilon
$$

These two equations, together with the definition $n_{t o t}(t)=n_{e}(t)+n_{t}(t)$ are equivalent to the continuity equation. Note that spatial and time dependence have been neglected in the generating term $G_{e x}$, and that the recombination term is given by $R=\alpha_{e r} n_{e}(t) n_{\text {tot }}(t)$. Since charges are excited homogeneously in the entire bulk the spatial dependence can be eliminated. Then using the continuity equation and inserting the recombination term gives

$$
\frac{\partial n_{t o t}(t)}{\partial t}=G(t)-R(t)=G_{e x}(t)-\alpha_{e r} n_{e}(t) n_{t o t}(t) .
$$

We can illustrate the connection between Eqs. (15) and (16) by adding Eq. (16) and Eq. (16) together - and recalling that when a trap distribution is present, we have $n_{\text {tot }}(t)=n_{e}(t)+n_{t}(t)$ - to obtain

$$
\frac{d n_{e}(t)}{d t}+\frac{d n_{t}(t)}{d t}=\frac{d n_{t o t}(t)}{d t}=G(t, z)_{e x}-\alpha_{e r} n_{e}(t)\left[n_{e}(t)+n_{t}(t)\right]+\alpha_{e t} n_{e}(t)\left[N_{t}-n_{t}(t)\right] .
$$

Equations (15) and (16) are the continuity equations with trapping, for this specific example. The form of Eq. (17) is not the most general form. Assuming some functional dependence of the DOS (and, therefore, the trapped states), one can write expressions for the filled trap state distributions $n_{t}(t)=\int_{0}^{\infty} n_{t}(\varepsilon, t) d \varepsilon$. Taking the derivative of Eq. (15) with respect to energy, including spatial dependence, and using the definitions for interaction coefficients and attempt to escape frequency discussed in Section 2.4, Eq. (2.6.7) can be written as:

$$
\frac{d n_{t}(\varepsilon, t)}{d t}=\alpha_{e t} n_{e}(t)\left[N_{t}(\varepsilon)-n_{t}(\varepsilon, t)\right]-\alpha_{t e} N_{e} \exp \left[-\frac{\varepsilon}{k T}\right] n_{t}(\varepsilon, t) .
$$

Equation (18) is finally the complete, one-dimensional, energy- and time-dependent trapping equation. Many of the prolific authors in the field of charge transport have used some form of this equation to describe carrier interactions with the DOS in HDIM for radiation induced conductivity, charge dynamics in electron irradiated materials and electrets. Excellent representations of the application of Eq. (2.6.7) in one form or another is given by the following 
list: non-penetrating radiation $\mathrm{RIC}^{51}$, non-penetrating radiation and parallel plate configurations ${ }^{52}$; non-penetrating radiation $^{53-62}$ photo-excited RIC systems ${ }^{63}$; and multiple configurations ${ }^{41}$.

We now return to the transport equations developed used to describe drift and diffusion in simple crystalline systems and extend these processes to include the effects of charge trapping. Using Poisson's equation, $\nabla \cdot \boldsymbol{D}=\rho_{c}$, the continuity equation, $\nabla \cdot\left(\mathrm{J}_{\mathrm{C}}+\mathrm{J}_{\mathrm{inj}}\right)=-\frac{\partial \rho_{\text {tot }}}{\partial \mathrm{t}}$, ther trapping equatons, and an explicit definition for the polarization, displacement, and diffusion current densities the following general transport equations can be found.

$$
\begin{gathered}
J_{t o t}(z, t)=q_{e} n_{e}(z, t) \mu_{e} F(z, t)+q_{e} D \frac{d n_{t o t}(z, t)}{d z}+\epsilon_{0} \epsilon_{r} \frac{\partial F(z, t)}{\partial t}+J_{p}(t) \\
\frac{\partial}{\partial z} F(z, t)=q_{e} n_{t o t} / \epsilon_{0} \epsilon_{r} \\
=G(z, t)_{e x}-\alpha_{e r} n_{e}(z, t) n_{h}(z, t)+\alpha_{e t} n_{e}(z, t)\left[N_{t}(z)-n_{t}(z, t)\right] \\
\frac{\partial n_{t o t}(z, t)}{d t}-\mu_{e} \frac{\partial}{d z}\left[n_{e}(z, t) F(z, t)\right]-q_{e} D \frac{\partial^{2} n_{e}(z, t)}{\partial n^{2}}= \\
\frac{d n_{t}(\mathrm{z}, \varepsilon, t)}{d t}=\alpha_{e t} n_{e}(z, t)\left[N_{t}(\mathrm{z}, \varepsilon)-n_{t}(\mathrm{z}, \varepsilon, t)\right]-\alpha_{t e} N_{e} \exp \left[-\frac{\varepsilon}{k T}\right] n_{t}(\mathrm{z}, \varepsilon, t)
\end{gathered}
$$

This set of equations covers a large class of carrier transport models in disordered materials whose applications range from Xerox copier element design and radiation effects in materials to descriptions of electron transport in power cable design ${ }^{64-69}$. The complexity of these equations, even in one dimension, is quickly becoming apparent. Manipulation of these equations for a specific application MUST be done with care. For examples of the application of these equations with spatial dependence see for penetrating radiation or RIC $\mathrm{R}^{70-74}$ Fig. 2 (c); for nonpenetrating radiation ${ }^{52}$; RIC and nonpenetrating radiation ${ }^{75-83}$ (see Figs. 2 (a) and (b); and for parallel plate configurations $^{84,85}$ (see Figs. 2 (c) and (d)). The complexity of the transport equations will decrease as a result of relevant assumptions based on each specific application.

There are two more phenomena, space charge limited current (SCLC) and dispersion, which can be described using this formalism. These are critical to more fully understand transport phenomena in HDIM. These two phenomena, SCLC and dispersion, are omitted because both require the DOS interaction picture, transport equations as described above, and further mathematical detail to describe their behavior which is beyond the scope of this paper. The interested reader should consult Ref 22.

It is critical to realize that the transport Esq. (19-23) cover both the charging of materials due to contacting electrodes, incident charged particle flux, incident photon fluxes, in addition to the decay of charge after the cessation of applied voltage(s) and incident particle flux. An investigation of the expressions for the decay of charge, current density, and surface or contacting electrode(s) can be found in Ref 22 in addition to many of the aforementioned authors but will not be discussed further here.

\section{G. Injection of charge}

A model of the interactions between the DOS in HDIM and macroscopic charge transport is only part of the story. Little consideration has been given to the generating term $G(z, t)$. It is critical to realize that the generating term is a function of the electronic state of the material. For example in the case of SEE, as charge is deposited or removed, the surface potential will change and so will the landing energy of incident charge. The theoretical problem of charge injection can therefore be addressed in two parts. First, we present a discussion of charge injection, that is the time evolution of the magnitude of the deposited charge. Second, we present a discussion of the depth to which charge will be injected, that is the location of the deposited charge layers as this changes with time. As outlined above, we will consider a simple parallel plate geometry system and then extend the discussion to injected charge configurations such as SEE. Taken together these describe nearly all the interface problems suggested by Fig. 2 and will allow for the final modification to the transport equations and our models.

\section{The Walden-Wintle Model of Charge Injection}

The injection problem has been addressed by many investigators. ${ }^{86-92}$ The work of Wilcox (Ref 86) and Walden (Ref 92) provide excellent descriptions of the problems involved. A very comprehensive, physically insightful, and 


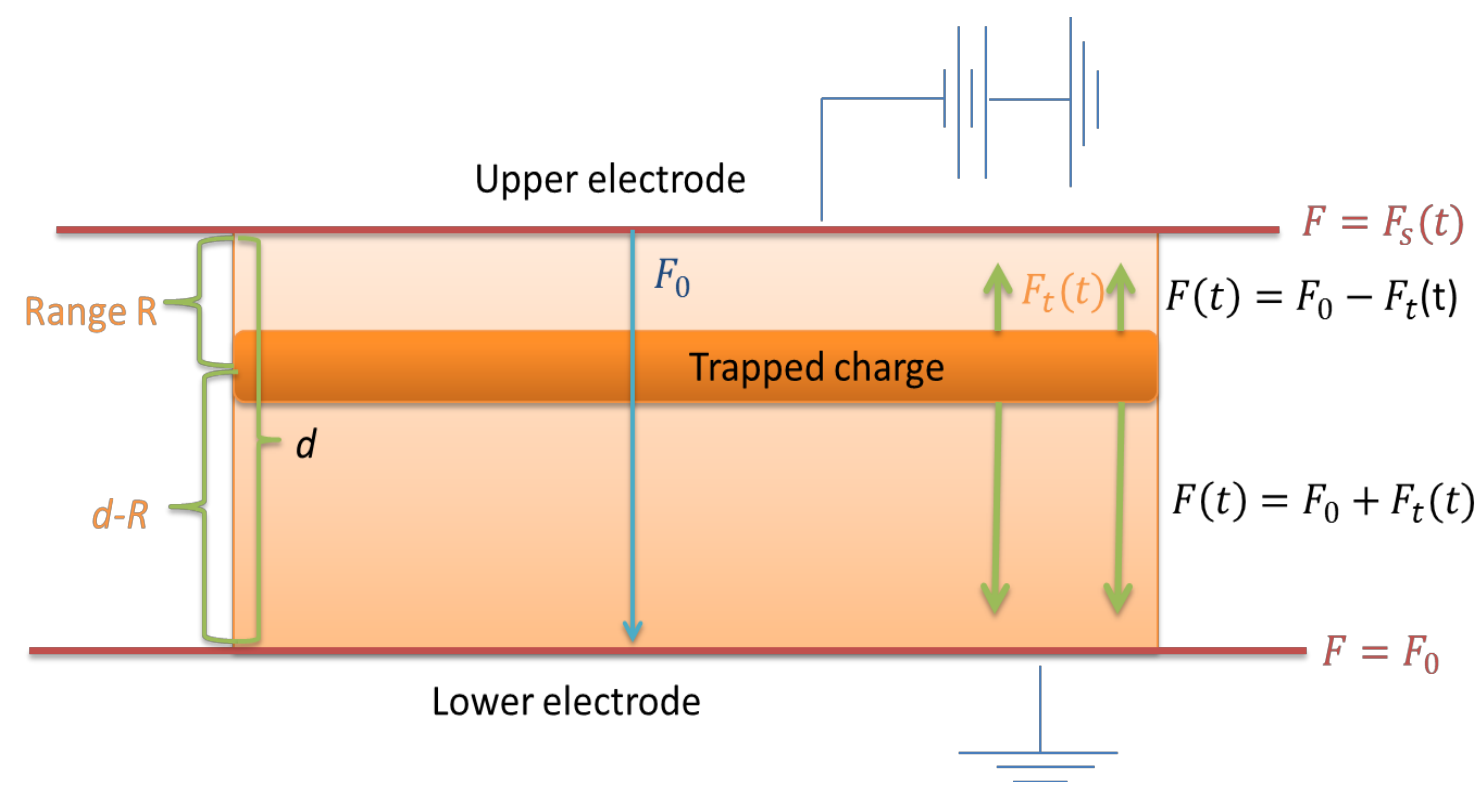

Figure 7. Basic electronic and field configuration of the CVC measurement system. All of the electric fields in the system are shown, except the polarization field: applied field, $F_{0}$, field due the trapped charge distribution; $F_{t}(t)$, surface field, $\boldsymbol{F}_{\boldsymbol{u p}}=F_{s}(t)=F_{0}-F_{t}(t), \boldsymbol{F}_{\text {down }}=F_{0}+F(t)$, field at the ground plane, $F_{0}$; and the superposition of fields at intermediate points, $F(t)$. The battery and ground are shown for reference. The range, $\mathrm{R}$, that the electrons penetrate into the sample, the distance between the trapped charge distribution and the rear, electrode $d-R$, and the sample depth, $\mathrm{d}$, are also shown.

easily applied theory was developed by Walden and later modified by Wintle. This semi-phenomenological theory characterizes the injection current density as a function of time, $J_{i n j}(t)$, at a metal-insulator interface. The model presented here is based on Walden's model, as modified by Wintle who provided a simple (nearly universal) form of the injection current density. Walden and Wintle showed for electrode injection that the behavior of $J_{i n j}(t)$ depends on the type of injection mechanism at work [e.g., Fowler-Nordheim, Schottky injection, space charge limited injection, and various tunneling mechanisms] in addition to the trapped charge distribution. ${ }^{84,85}$ The model is extended to include electron beam injection here. ${ }^{24}$ In what follows, we give a brief outline of the derivation for the injection current density function.

Consider the electric field at the surface of the HDIM, $F_{S}(t)$, that must change as the trapped charge distribution builds up. We assume for simplicity that only electrons are injected into the material through a metal-insulator interface to a uniform depth, $R$; this is quite different from a simple capacitor model where we assume positive charges are at one side where the potential is high (from which the electric field extends) and negative charges at the other side (where the field lines terminate) where the potential is low. While this simple capacitor model is still useful, we now have an injected electron distribution at or near the interface of the material. Consider Fig. 7 in which the applied field, $F_{0}$, the surface field, $F_{S}(t)$, the field due to trapped charge, and the superposition of internal fields, $F(t)$, are shown. This is consistent with Fig. 3 for the case where $J_{b}=J_{\text {emit }}=0, J_{u p}=J_{\text {inj }}, V_{\text {ext }}=V_{b}=$ 0 , and $F_{0}$ resutls from $V_{\text {bias }}$. Note, that $F(t)$ is different from the surface field, but the spatial dependence has been omitted for simplicity. At time, $t=0, F_{S}(t)$ must be equal to the applied field, $F_{0}$, but as time goes on $F_{S}(t)$ must be reduced by the electric field due to the trapped charge distribution, $F_{t}(t)$. In a general way, we can write the following equation for the electric field at the surface

$$
F_{s}(t)=F_{0}-F_{t}(t)
$$


Since, in practice, we know the applied field, only $F_{t}(t)$ is required to estimate this relation. From Gauss's law, Eq. (1), we can write

$$
\frac{\partial}{\partial \mathrm{z}} F_{t}(z, t)=q_{e} n_{t}(z, t) / \epsilon_{0} \epsilon_{r}
$$

If we assume the charge distribution at depth $\mathrm{R}$ is the product of a time-dependent function and a time-independent 1D spatial function, which is uniform from $z=0$ (the collecting electrode at ground) to $z=(d-R)$, then the spatial dependence of the trapped charge distribution can be approximated by a step function, $n_{t}(z, t)=$ $n_{t}(t) \theta(z-(d-R))$. Note that PEA measurements reveal that the spatial dependence in the $z$ direction of the charge distribution is closer to a Gaussian or exponential distribution; extension of the uniform charge distribution approximation is not addressed here. Under these conditions, we can integrate Eq. (25) with respect to $\mathrm{z}$ from $z=$ 0 to $z=d-R$ to obtain an expression for the field due to the trapped charge distribution

$$
F_{t}(t)=q_{e} n_{t}(t)(d-R) / \epsilon_{0} \epsilon_{r}
$$

Using Eqs. (24) and (26), a new equation for the electric field at the surface of the sample can be obtained

$$
F_{s}(t)=F_{0}-q_{e} n_{t}(t)(d-R) / \epsilon_{0} \epsilon_{r} .
$$

Equation (27) gives us both the electric field at the surface of the sample and the potential at the surface of the sample, since $F(t)=-\nabla \cdot V(t)$. Eq. (27) suggests that if all the charge were exactly on the surface of the sample (e.g., $R=0$ ) then there would be a trapped charge field. This result is consistent with a DOS at (or near) the surface of the material acting to trap charge and create a counter field, $F_{t}(t)$; it is also consistent with the requirement that all the trapping occurs at or near the surface of the material (a result consistent with PEA measurements). Note also, that when $R \geq d$ for penetrating radiation, the field goes to $F_{0}$, suggesting that the charge distribution is outside of the material; this is the expected result. The field between the trapped charge distribution and the rear electrode is the applied field plus the field due to the trapped charge distribution and is the field that drives the current measured at the rear electrode. The current model can be improved by including, the spatial dependence of the electric field, with the inclusion of a spatially-dependent susceptibility or dielectric function to better model the spatial behavior at the surface and rear electrode.

Equation (27) gives a first - order approximation for the electric field at the surface of the material, which must be directly tied to the currents flowing in the sample. For the configurations described by Fig 2 (b) and Fig. (7), we seek an expression for the current density measured at the rear electrode. We will use the theory of Walden and Wintle to develop an expression for the injection current density using Eq. (27). Walden addresses the problem of finding an expression for the surface potential based on the injection behavior of charge at the surface and subsequent trapping of that charge. We outline the derivation of Walden below.

Essentially, a relationship between the injection profile caused by the application of an electric field and the effects caused by the electronic nature of the metal-insulator interface combined with the electric field generated by the trapped charge given by Eq. (27) is required. First, consider how the electric field changes at the surface of the material,

$$
\frac{d F_{S}(t)}{d t}=-\frac{q_{e}(d-R)}{\epsilon_{0} \epsilon_{r}}\left[\frac{d}{d t} n_{t}(t)\right] .
$$

The total charge that will pass through the surface to the rear electrode must be $q_{e} d n(t) / d t$, where $n(t)$ is the total number of electrons that passes through the surface as a function of time. Recall that $n(t)$ is the volume density of electrons and that the current density is defined as charge per unit area per unit time; with this in mind, we can write the current density that passes through the surface of the insulator as $J_{i n j}(t)=-q_{e} d \frac{d n(t)}{d t} ; d$ is the depth of the sample as the current passing through the surface must be the current that will eventually traverse the sample. Equation (28) does not explicitly contain the total current, but it is quite clear that some fraction of the total current must be trapped in the insulator. Applying the chain rule to Eq. (28)., $\frac{d n(t)}{d t} \rightarrow\left\{\frac{d n(t) \cdot d n_{t}(t)}{d n_{t}(t) \cdot d t}\right\}$, and Eq. (28) and the relation for the current density at the interface, taken together become 


$$
\frac{d F_{S}(t)}{d t}=-\frac{q_{e} d_{1}}{d \epsilon_{0} \epsilon_{r}} J_{i n j}(t) \frac{d n_{t}(t)}{d n(t)}
$$

An expression for the change in the trapped charge distribution to the total charge, $\frac{d n_{t}(t)}{d n(t)}$, is needed. This is a very useful relation in of itself. For example, in the secondary electron emission chamber, the USUMPG can measure the total charge, surface injection current density, and the resulting surface potential to determine the behavior of the injection current and ratio of trapped to total charge; an extended discussion of the relationship between Eq. (29), surface potential, and secondary electron emission is given in Ref 22. Equation (29) is used below to directly tie electron beam injection modes to the Walden-Wintle formalism.

Walden makes an experimentally based phenomenological assumption to extend Eq. (29); the form of the ratio of total electrons to trapped electrons is assumed to be a power law of the injection current density, $J_{i n j}(t)$, to the initial current density, $J_{0}$, of the uncharged sample. Using this assumption, one gets

$$
\frac{d n_{t}(t)}{d n(t)}=\left(\frac{J_{i n j}(t)}{J_{0}}\right)^{n-1}
$$

Equation (30) is a simple power law relationship in which total trapping is given when $n=1$ and partial trapping for $n>1$; increasing $n$ implies stronger trapping. A detailed analysis of the physical roots of Eq. (30) and its validity have been given in Ref 91. Using Eqs. (28) and (30) an expression that describes the relationship between the trapped-electron distribution-dependent electric field, the injection current at the surface, and time is found to be

$$
t=-\frac{\epsilon_{0} \epsilon_{r} d}{(d-R) J_{0}} \int_{F_{S}(0)}^{F_{S}(t)}\left(\frac{J_{i n j}\left(\hat{F}_{S}\right)}{J_{0}}\right)^{-n} d \hat{F}_{S} .
$$

Equation (31) is Walden's central result; it allows the time dependence of any surface field-dependent function to be found. Walden explores four major types of electrode injection behavior describe in terms of the dependence of injection current on surface field: (i) Pooler-Frenkle, (ii )Fowler-Nordheim, (iii) pure exponential, and (iv) power law. Table 2 summarizes the mathematical relations for each of the injection behaviors. Wintle (Ref 90) has explored these injection types and found essentially that all yield a similar form for $J_{i n j}(t)$. As an example, the simplest derivation for the current density at the surface is found by using the power law form of $J_{\text {inj }}\left(\hat{F}_{s}\right) / J_{0}=$ $\left(F_{S}(t) / F_{0}\right)^{p}$ with $p$ as constant greater than one. Using this power law form and Eq. (31), the following form for the injection current density is easily found to be

$$
J_{\text {inj }}(t)=J_{0}\left(1+t / \tau_{\text {onset }}\right)^{-\frac{p}{n p-1}}
$$

Remember that this is the injected current density, not the measured current density at the rear electrode. Further, this expression relates only to systems with a power law like injection behavior and where the injection current density is known. The product of $n p \geq 1$ is always greater than or equal to one since-by definition - both are independently greater than or equal to one. The variable, $\tau_{\text {onset }}$, is a characteristic onset time for the injection current density type, not to be confused with a decay time or $\tau_{\text {transit }}$. In practice, the onset time describes the time at which the current density at the metal-HDIM interface will begin to diminish due to trapping processes and field effects.

Wintle generalizes the resulting injection current density at the interface, providing a simple model, which can be used to overcome the coupling between bulk trapping and the resulting injection current density without a detailed consideration of the metal insulator interface effects. It turns out, for most injection phenomena - when moderate to strong trapping is considered - the injection current density can be well characterized by

$$
J_{\text {inj }}(t)=J_{0}\left(1+t / \tau_{\text {onset }}\right)^{-m}+J_{\text {sat }}
$$

Here, $J_{0}$ is the initial injection current density at time $t=0, J_{\text {sat }}$ is the equilibrium injection current density as some time, $t \gg \tau_{\text {onset }}$, and $m$ is a generalized power that is dependent on the strength of the trapping of injected electrons [see Eq. (30)] and the strength of the field-dependent injection rate [see Eq. (31)] (to relate Wintle's results to Walden's formalism, note $m \equiv 1 / n)$. For HDIM, $J_{\text {sat }}$ will not be observed until the equilibrium current occurs. The expression of the injection current given by Eq. (33) is easily applied to capacitor type models in which there are injecting electrodes given by Fig 2. (b). ${ }^{22}$

Using the transport equations to develop a simple model for the charging of an insulator due to injected electrons can now be approached. Equation (23) provides a direct connection between the injection current density and the 
Table 2. List of injection barrier types. Five major types of injection barriers for electrode injection (Walden, 1972) and three types of electron beam injection (Wilson, 2013) are listed. $F_{S}$ is the instantaneous field at the injection electrode or charge layer. $F_{c i}$ are scaling fields and $C_{i}$ and $n_{i}$ are constants, unique for each model and material. $p$ is a power law constant and $\chi$ is the Electron affinity. $Y_{o}\left(\varepsilon_{b}\right)$ is the initial (uncharged) total electron yield, $R\left(\varepsilon_{b}\right)$ is the range, $d$ is the sample thickness, and $V_{s}$ is the surface bias voltage.

\begin{tabular}{|c|c|c|}
\hline Injection Barrier Type & Injection Current, $J_{i n j}(F)$ & Time Constant, $\tau_{o}$ \\
\hline \multicolumn{3}{|l|}{ Surface Electrode Injection } \\
\hline $\begin{array}{l}\text { Poole-Frenkel } \\
\text { (Schottky/thermionic emission) }\end{array}$ & $C_{1} F_{S} e^{\left(F_{S}+F_{c 1}\right)^{1 / 2}}$ & {$\left[\frac{n_{1} \epsilon_{o} \epsilon_{r} d}{J_{0}(d-R)}\right] 2\left(\frac{V_{s}}{d}\right)^{1 / 2} F_{c 1}^{1 / 2}$} \\
\hline $\begin{array}{l}\text { Fowler-Nordhiem } \\
\text { (Tunneling-type field emission) }\end{array}$ & $C_{2} F_{s}^{2} e^{-\left(F_{c 2} / F_{s}\right)}$ & {$\left[\frac{n_{2} \epsilon_{o} \epsilon_{r} d}{J_{0}(d-R)}\right]\left(\frac{V_{s}}{d}\right)^{2} F_{c 2}^{-1}$} \\
\hline Simple exponential & $C_{3} e^{\left(F_{S} / F_{c 3}\right)}$ & {$\left[\frac{n_{3} \epsilon_{o} \epsilon_{r} d}{J_{0}(d-R)}\right] F_{c 3}$} \\
\hline $\begin{array}{l}\text { Constant } \\
\left.\text { (Exponential with } F_{c} \rightarrow \infty\right)\end{array}$ & $C_{4}$ & $\tau_{o} \rightarrow \infty$ \\
\hline Power law $\mathrm{p}>>1$ & $J_{0}\left(F_{s} / F_{c 5}\right)^{p}$ & {$\left[\frac{n_{5} \epsilon_{o} \epsilon_{r} d}{J_{0}(d-R)}\right] \frac{1}{p}\left(\frac{V_{s}}{d}\right)$} \\
\hline \multicolumn{3}{|l|}{ Electron Beam Injection } \\
\hline Penetrating beam & 0 & $\tau_{o} \rightarrow \infty$ \\
\hline $\begin{array}{l}\text { Positive Charging } \\
(\text { Total Yield }<1)\end{array}$ & $J_{o}\left[1-Y_{o}\left(\varepsilon_{b}\right)\right] e^{-\left(F_{s} / F_{c 6}\right)}$ & {$\left[\frac{n_{6} \epsilon_{o} \epsilon_{r} d}{J_{0}\left[1-Y_{o}\left(\varepsilon_{b}\right)\right]\left(d-\frac{1}{2} R\right)}\right] F_{c 6}$} \\
\hline $\begin{array}{l}\text { Negative Charging } \\
\text { (Total Yield }<1 \text { ) }\end{array}$ & $J_{o}\left\{1-Y_{o}\left(\varepsilon_{b}\right)\left[\frac{\chi^{2}\left[3 F_{s}\left(d-\frac{1}{2} R\right) q_{e}+\chi\right]}{\left[3 F_{s}\left(d-\frac{1}{2} R\right) q_{e}+\chi\right]^{3}}\right]\right\}$ & Numerical solution required \\
\hline
\end{tabular}

trapped charge distribution. For simplicity the spatial and thermal dependence in Eq. (23) are ignored and a relationship between the interaction coefficient, number of electrons in the $\mathrm{CB}$, and the injected current density is given by $\alpha_{e t} n_{e}(t)=s_{c} J_{i n j}(t) / q_{e}$. Using these assumptions Eq. (23) becomes

$$
\frac{d n_{t}(t)}{d t}=\frac{s_{c} J_{s}(t)}{q_{e}}\left[N_{t}-n_{t}(t)\right]
$$

Equation (34) is a differential equation that predicts the change in the trapped charge distribution, $n_{t}(t)$, as a function of time, total DOS, $N_{t}$, and a surface injection current density, $J_{i n j}(t)$. This equation has been used by many authors to investigate a wide array of charging and ESD phenomena. A few key authors are listed for the interested reader: electron emission, ${ }^{43,52,94,95}$ capacitive methods; ${ }^{88}$ capacitive methods and ESD $;{ }^{87}$ charging by radiation, ${ }^{18}$ impact ionization and transport $;{ }^{96}$ capture and emission; ${ }^{97}$ ESD and charge trapping; ${ }^{98}$ trapping and detrapping dynamics, ${ }^{98}$ and photoconduction. ${ }^{99}$ Equation (34) can be solved to obtain an expression for the current density at the rear electrode and surface potential due to injection for the predicted trapped charge distribution:

$$
n_{t}(t)=N_{t} \exp \left[\frac{-s_{c} \int_{0}^{t} J_{i n j}(t) d \tilde{t}}{q_{e}}\right] \int_{0}^{t} \exp \left[\frac{-s_{c} \int_{0}^{t} J_{i n j}(\tilde{t}) d \tilde{t}}{q_{e}}\right] \frac{s_{c}}{q_{e}} J_{i n j}(\hat{t}) d \dot{t}
$$

Equation (35) describes the number of trapped electrons as a function of time that results from some injection current density and DOS. In order to solve this equation, one needs an expression for the injection current density, $J_{\text {inj }}(t)$. 
The first step in creating a complete description of the charge build up that includes dispersive behavior is to extend Eq. (34) to include the thermal energy dependence. This is easily done using the transport equations Eqs. (16) and (15)

$$
\frac{d n_{t}(t)}{d t}=\frac{s_{c} J_{i n j}(t)}{q_{e}}\left[N_{t}-n_{t}(t)\right]-v_{t e} \int \exp \left[-\frac{\varepsilon}{k_{b} T}\right] n_{t}(\varepsilon, t) d \varepsilon .
$$

In Eq. (36), $N_{t}=\int N_{t}(\varepsilon) d \varepsilon$ is the total DOS and $v_{t e}$ is a factor that describes the attempt-to-escape frequency of a charge trapped in the DOS. The last term on the RHS determines the release rate of trapped electrons at a specific energy integrated over the width of the DOS distribution. Inclusion of this term is equivalent to having a spread in the release times of the trapped electrons (i.e., there is a dispersion in the release times and, therefore, the electron transport). When dispersive transport is significant the inclusion of the last term is necessary. In general, dispersive transport occurs on timescales much longer than are required to charge up the sample in many typical experiments..

Equation. (33) can be extended to include experimental configurations in which the charge is injected by means of an charged particle beam (see Fig 2). The meaning of $J_{0}$ for charged beam injection is not the same as it is for electrode injection. For charge beam injection, the number of electrons injected into the sample is proportional to the number of electrons from the incident electron beam, but this must be corrected to include the effects of electron emission and reattraction of electrons to a charged surface through a charge dependant electron yield, $Y_{o}$. When nearly all the electrons are injected into or pass though the sample the yield is zero, and for the case where as many electrons are emitted as incident on the sample the yield is one. That is, for charge beam injection the initial current density must be scaled by the total electron yield. ${ }^{25}$

In terms of Walden's formalism for charge injection (refer to Eq. (30) for electron beam injection with no emission, $\left[d n_{t} / d n\right]=1$ corresponds to a non-penetrating beam and $\left[d n_{t} / d n\right]=0$ corresponds to a penetrating beam. In general for emission, the incident charge density is equal to the trapped charge density minus the emitted charge density; that is in terms of yield

$$
\left[d n_{t} / d n\right]=\left[1-Y_{o}\left(t ; E_{b}\right)\right]
$$

and from Eq. (30) the instantaneous injection current is

$$
J_{i n j}(t)=J_{o}\left[1-Y_{o}\left(t ; E_{b}\right)\right]
$$

with $m=1 / 2$. Also note that for $\mathrm{Y}>1$, the injection current has the opposite sign of the initial current, as should be the case.

As a first order approximation, we assume a static electron emission independent of time and accumulated charge. Then $J_{0} \rightarrow J_{b}\left(1-Y_{o}\right)$, where $J_{b}$ is the current density of the incident electron beam. The saturation current has a similar meaning in this context but typically will occur on much shorter time scales. Making the correction to the injection current density function, Eq. (33), for static emission becomes

$$
J_{s}(t)=J_{b}\left(Y_{o}-1\right)\left(1+\frac{t}{\tau_{\text {onset }}}\right)^{-m}+J_{\text {sat }} \rightarrow J_{b}\left(\sigma_{\text {yeild }}-1\right)+J_{\text {sat }} .
$$

For the dynamic emission model with yield dependant on accumulating charge in the negative charging region where $\sigma_{\text {yeild }}<1$, based on Thomson ${ }^{47}$ and Hoffmann's ${ }^{18,25}$ model for yield as a function of accumulated charge,

where

$$
J_{\text {inj }}(t)=J_{o}\left\{\left[1-Y\left(E_{b}\right)\right] e^{-\left(t / \tau_{\text {onset }}\right)}\right\}
$$

$$
\tau_{\text {onset }}=\left[\frac{n_{6} \epsilon_{o} \epsilon_{r} d}{J_{0}\left[1-Y_{o}\left(\varepsilon_{b}\right)\right]\left(d-\frac{1}{2} R\right)}\right] F_{c 6}
$$

For the dynamic emission model with yield dependant on accumulating charge in the positive charging region where $\sigma_{\text {yeild }}>1$, based on Wilson ${ }^{114}$ and Hoffmann's ${ }^{18,25}$ model for yield and charge re-attraction as a function of accumulated charge, 


$$
J_{i n j}(t)=J_{o}\left\{1-Y_{o}\left(\varepsilon_{b}\right)\left[\frac{\chi^{2}\left[3 F_{s}\left(d-\frac{1}{2} R\right) q_{e}+\chi\right]}{\left[3 F_{s}\left(d-\frac{1}{2} R\right) q_{e}+\chi\right]^{3}}\right]\right\}
$$

where the term in square brackets is the ratio of emitted secondaries reattracted to the positively charged surface to the total number of secondaries emitted. Table 2 summarizes the mathematical relations for charge beam injection behaviors.

The interested reader should consult (Ref 90) and (Ref 92) for a complete development of all the ideas discussed in this section. These results must be worked out in more detail for each type of metal-insulator interface, applied field, temperature dependence, and type of injection mechanism. For each specific application and experimental configuration, it is critical that the use of Eq. (30) be validated and understood before the application of the theory to develop a complete picture of the injection current density dependence on the applied field, electric field due to the trapped charge distribution, and time. With that said, Eq. (33) has been applied successfully in a number of experimental trials; $;^{86,88,90-92}$ note, however, that (Ref 88 ) uses a physically unjustified form of the equation.

\section{Injection Range}

In our development of Eq. (27) an injection range was suggested for contacting electrodes. This value is so small compared to the sample thickness for typical electrode injection configurations as to be considered negligible. However, for charged beam injection this is not the case. The total electron yield, $\sigma_{\text {yeild }}(\varepsilon, t)$, is a function of incident beam energy and therefore the penetration depth of an incident charged particle into the sample, which can be appreciable compared with $d$ at higher energies.. A study of the range as a function of incident beam energy is therefore critical for our understanding of SEE and related phenomena. The range, $R\left(\varepsilon_{\text {inc }}\right)$, or maximum distance an electron of a given incident energy can penetrate through a material before all kinetic energy is lost and the electron comes to rest, is a common way to parameterize electron interactions with materials. The energy-dependant range is commonly used to predict the charge distribution of deposited electrons in materials and to model SEE. It is also used to predict the distribution of energy deposited by incident electrons as they traverse a material; this distribution is further used to model RIC. As noted above (see Fig. 2), the ratio of the range to sample thickness determines the type of experimental configuration. This in turn guides the choice of appropriate simplifying approximations to use and the subsequent development of the appropriate transport equations for a wide array of conducting, semiconducting and insulating materials with a minimal number of fitting parameters.

The desired range expression can be developed by merging well known semi-empirical models for the interaction of electrons with materials in different energy regimes, employing the continuous-slowing-down approximation (CSDA). In the CSDA, the rate of energy loss, $d \varepsilon / d z$ (or total stopping power, $S$ ), at every position along the penetration path is assumed constant; variations in energy-loss rate with energy, $\varepsilon$, or penetration depth are neglected. For a given incident energy, $\varepsilon_{i n c}$, the CSDA range is obtained by integrating total stopping power over the full penetration depth ${ }^{100,101}$ such that

$$
\varepsilon_{\text {inc }}=\int_{0}^{R\left(\varepsilon_{i n c}\right)}(d \varepsilon / d z) d z
$$

In the CSDA with a constant energy-loss rate,

$$
d \varepsilon / d z \equiv S_{C S D A}\left(\varepsilon_{i n c}\right)=\varepsilon_{i n c} /_{R}=\bar{\varepsilon} / \bar{\lambda}=\varepsilon_{\min } / \lambda_{\min }
$$

Here $\bar{\varepsilon}$ is equal to mean energy lost per collision occurring at mean free path $\bar{\lambda} \equiv \lambda_{I M F P}(\bar{\varepsilon})$, and $\varepsilon_{\min }$ is the energy at the minimum in the inelastic mean free path curve at $\lambda_{\min } \equiv \lambda_{I M F P}\left(\varepsilon_{\min }\right)$, Ref 104 .

At higher incident energies, the non-relativistic Bethe-Joy range expression based on the Bethe stopping power formula ${ }^{105}$ has been extended by Joy and Luo ${ }^{106}$ and Tanuma ${ }^{107}$ to provide good semi-empirical models for the range. Tabulated values of the electron ranges at high energies using the CSDA can be found in the NIST ESTAR database $^{108}$ spanning incident energies from $\varepsilon H I \sim 20 \mathrm{keV}$ up to $\sim 1 \mathrm{GeV}$. Computer models such as SREM ${ }^{109,110}$ and NUMIT2 $2^{11,112}$ provide simulations of the range and the spatial distribution of electrons deposited within the material. However, it is important to be able to extend range models to lower incident energies. Use of the CSDA and models and measurements of the inelastic mean free path (IMFP) provide a means to do this. The NIST IMFP database ${ }^{113}$ has tabulated values and semi-empirical fits for the IMFP ${ }^{104}$, which are valid for energies from $\sim 30 \mathrm{eV}$ to $\varepsilon_{L O} \sim 1 \mathrm{keV}$. 
Wilson and Dennison ${ }^{44,114}$ used the CSDA, to develop an empirical fit to the range with a continuous, simple, composite, analytic formula - with a single free parameter, termed the effective number of valence electrons, $N_{V}^{\text {eff }}$ - to approximate the range $\left(10^{-9} \mathrm{~m}\right.$ to $\left.10^{-2} \mathrm{~m}\right)$ over an extended energy span $(<10 \mathrm{eV}$ to $>10 \mathrm{MeV})$. Agreement with available databases of electron interactions are within $<20 \%$ for a broad set of conducting, semiconducting, and insulating materials. The problem was broken into three parts according to energy of the incident electron: a high energy range for $\varepsilon_{i n c}>\varepsilon_{L O} \equiv 1 \mathrm{keV}$; a mid-energy range for $\bar{\varepsilon}<\varepsilon_{i n c}<\varepsilon_{L O}$; and a low energy range for energies $\varepsilon_{\text {inc }}<\bar{\varepsilon}$. The final result is a continuous composite analytic approximation to the range, spanning from $<10 \mathrm{eV}$ to $>10 \mathrm{MeV}$ :

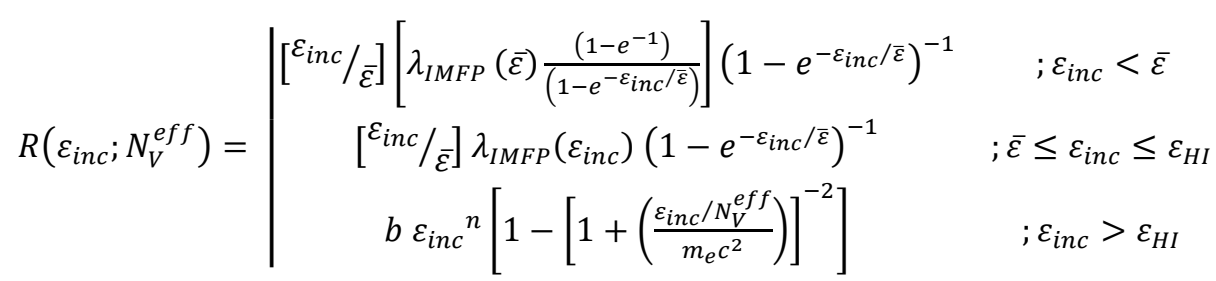

The high energy term takes the form of numerous power law models of the stopping power that have been developed with $0.35 \leq \mathrm{n} \leq 0.67$ (Reimer, 2000); physical constraints require $0 \leq n \leq 1$. The non-relativistic Bethe-Joy range expression based on the Bethe stopping power formula ${ }^{105}$ has been extended by Joy and Luo ${ }^{106}$ and Tanuma $^{107}$, with the addition of a fixed empirical constant, $k=0.8$. This is used to fit the data up to $\sim 10^{5} \mathrm{eV}$, above which a relativistic correction ${ }^{44}$ becomes significant ${ }^{115}$. The stopping power exponent $n$ and proportionality constant $b$ can be expressed in terms of $N_{V}^{e f f}$ by matching the slope and magnitude of the approximate power law formula, to the Bethe-Joy and mid-energy range expression, respectively. This leads to an expression for the stopping power exponent

$$
n\left(N_{V}^{e f f} ; \rho_{m}, M_{A}, \varepsilon_{\text {gap }}\right)=\ln \left\{\frac{\ln \left[\sqrt{\frac{e}{2}}\left(\frac{\varepsilon_{H I} / N_{V}^{e f f}}{\bar{\varepsilon}}+k\right)\right]}{\ln \left[\sqrt{\frac{e}{2}}\left(\frac{\varepsilon_{L O} / N_{V}^{e f f}}{\bar{\varepsilon}}+k\right)\right]}\right\}\left[\ln \left[\frac{\varepsilon_{L O}}{\varepsilon_{H I}}\right]\right]^{-1}+1
$$

The magnitude of the high energy range expression, Eq. (45), is normalized to the mid-energy expression at $E_{L O}$, by setting

$$
b\left(N_{V}^{e f f} ; \rho_{m}, M_{A}, \varepsilon_{\text {gap }}\right)=\frac{\varepsilon_{L O} O^{1-n} \bar{\lambda}\left(1-e^{-\varepsilon_{L O} / \bar{E}}\right)^{-1}}{\bar{\varepsilon}\left(\left[1-\left[1+\left(\frac{\varepsilon_{L O} / N_{V}^{e f f}}{m_{e} c^{2}}\right)\right]^{-2}\right]\right)}
$$

Direct extrapolation of the range from the ESTAR data to lower energies is not valid for energies comparable to the atomic electronic structure, typically a few $\mathrm{keV}$ and below, because the discrete energy nature of the collisions becomes important. However, a simple extension of the CSDA to lower energies can relate the range to the electron IMFP $^{104}$. The stopping power in Eq. 40 is assumed equal to the total energy lost $\left(\varepsilon_{\text {inc }}\right)$ divided by the total distance traveled (range, $R\left(E_{\text {inc }}\right)$ ). This is set equal to the mean energy lost per collision, $\bar{E}$, divided by the mean distance traveled per collision all times the probability that a collision occurs, $\left(1-e^{-R / \bar{\lambda}}\right)=\left(1-e^{-\varepsilon_{\text {inc }} / \bar{E}}\right)$. For $\bar{\varepsilon}>\varepsilon_{\text {inc }}>\varepsilon_{H I}$, $\lambda_{\text {IMFP }}\left(\varepsilon_{\text {inc }}\right)$ is assumed to be given by the TPP-2M formula used in conjunction with the NIST IMFP database ${ }^{113}$ : A reasonable approximation for $\bar{E}$ is the geometric mean of the effective plasmon energy, $\varepsilon_{p}^{\text {eff }}$, and the bandgap energy $^{103}, E_{\text {gap }}$, times an empirically determined factor ${ }^{102}$ of 2.8 :

$$
\bar{\varepsilon}=2.8\left[\left(\varepsilon_{p}^{e f f}\right)^{2}+\left(\varepsilon_{\text {gap }}\right)^{2}\right]^{1 / 2}=2.8\left[\hbar^{2}\left(N_{V}^{e f f} N_{A} \rho_{m} q_{e}{ }^{2} / m_{e} \varepsilon_{0} M_{A}\right)+\left(\varepsilon_{\text {gap }}\right)^{2}\right]^{1 / 2}
$$

$q_{e}$ and $m_{e}$ are the electron charge and rest mass, $\hbar$ is the reduced Planck's constant, $\varepsilon_{0}$ is the permittivity of free space, $N_{A}$ as Avogadro's number, $M_{A}$ is the atomic weight, and $\rho_{m}$ is the mass density. While $\varepsilon_{\text {gap }}$ may be considered an additional fitting parameter for semiconductors and insulators, its effect on $R$ is minimal, causing primarily a vertical shift in the range curve within $2 \%$ using acceptable band gap energies. Thus, $\varepsilon_{\text {gap }}$ can be treated essentially 


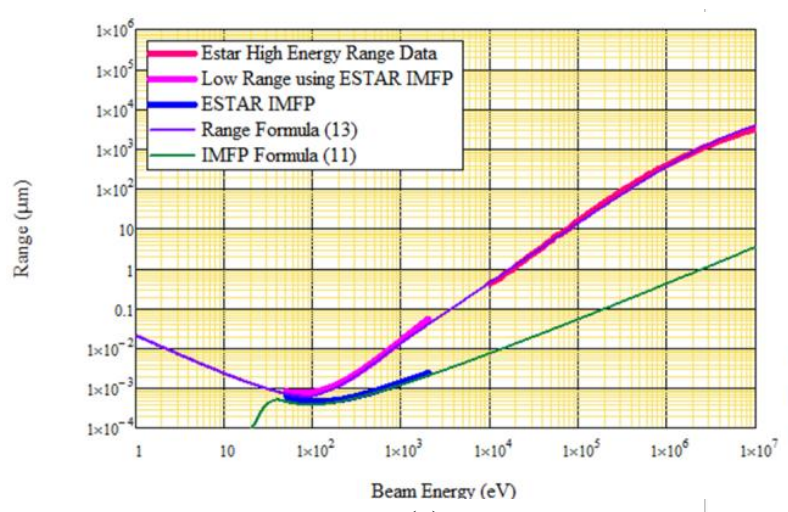

(a)

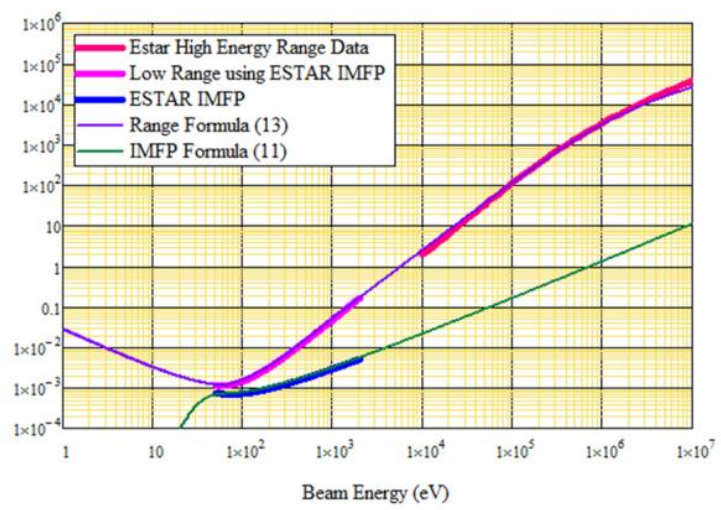

(b)

Figure 8. Comparison of range formula with known data. Comparison of the range formula for (a) Au with $\mathrm{N}_{\mathrm{v}}=$ 12.0 . and (b) polyimide (Kapton ${ }^{\mathrm{TM}}$ ) with $\mathrm{N}_{\mathrm{v}}=4.1$.

as an additional tabulated material constant—such as $M_{A}$ and $\rho_{m}$ are-derived from independent optical measurements.

To calculate the range for $\varepsilon_{i n c}<\bar{\varepsilon}$, we assume in the CSDA that: (i) the energy lost per low energy collision is constant and equal to the mean excitation energy, $\bar{\varepsilon}$; (ii) the IMFP is constant and equal to the IMFP at the mean energy loss or $\lambda_{I M F P}(\bar{\varepsilon})=\bar{\lambda}$; and (iii) the probability that an electron undergoes one such inelastic collision falls off as $R / \bar{\lambda}\left(e^{-R / \bar{\lambda}}\right)=\varepsilon_{b} / \bar{\varepsilon}\left(e^{-\varepsilon_{b} / \bar{\varepsilon}}\right)$. This simple, mean-field, low energy approximation avoids the unusual asymptotic behavior exhibited by the TPP-2M expression at energies below $\bar{\varepsilon}$, Ref 113. The resulting expression is consistent with a universal curve of electron IMFP versus kinetic energy ${ }^{117}$ observed for a wide range of materials ${ }^{118}$, that is consistent with a simple free electron gas model of valence electrons in the material ${ }^{116}$.

Figure 8 show best fits to data for the conductor Au and insulator Kapton. This new model provides a final piece to complete the extension of the transport equations to electron beam injection experimental systems described in Fig 2.

Given an outline of the conduction processes, band structure, microscopic and macroscopic transport, transport equations, and the extension of the trap controlled transport equations to include injection of charged carriers into the material we now present an application of the theory that connects these ideas to surface potential models for secondary electron beam injection.

\section{Surface Potential Models}

The experimental configurations outlined in Fig. 2 have the injection current $\left(J_{b}\right)$ and beam energy $\left(E_{b}\right)$ or electrode voltage $\left(V_{\text {bias }}\right)$ as inputs. The measured responses to be modeled here are the various output currents and the surface voltage $\left(V_{s}\right)$ shown in the generalized experimental configuration ${ }^{24,44}$ shown in Fig. 3. We assume a simple dielectric slab or parallel plate capacitor geometry with a dielectric material of thickness, $d$, above a grounded rear electrode. This assumption follows for thin film samples and charged-particle-beams whose cross sectional area is approximately the sample area if the lateral extent of the deposited charge layers is uniform and is much larger than $d$, so edge effects and fringing fields can be neglected. Under this assumption, the charge deposited can be thought of as a simple planar sheet, which leads to a 1D model of electric transport.

$\mathrm{Sim}^{22}$, Hodges ${ }^{24}$, and Wilson ${ }^{44}$ use the general geometry in Fig. 3 to develop detailed explicit equations for the electric field and electric potential everywhere inside the dielectric, as well as the surface voltage, grounded rear electrode current, front electrode current, and emission current by modeling the system in terms of the superposition of uniform electric fields extending in both directions produced by a series of 2D charge plane double layers. They consider successively more complex models, beginning with no charge emission and no charge redistribution or dissipation through conduction. Cases for static, dynamic and general emission through $J_{\text {emit }}$ are then considered. Finally, charge dissipation is incorporated through a potentially time-dependant expression for the conductivities above $(0<z<R)$ and below $(R<z<D)$ the embedded charge layer $\left(\sigma_{u p}(t)\right.$ and $\sigma_{\text {down }}(t)$, respectively). Cases are considered for: (i) no dissipation (zero conductivites), (ii) static dissipation (time-independent conductivities), (iii) dynamic charge dissipation (time-dependent conductivities and the additional possibility of the motion of the planar embedded charge layer, and (iv) dynamic charge dissipation with more general time-dependant conduction that allows for the evolution of the spatial distribution of the embedded charge. They found that 
$\mathrm{V}_{\mathrm{d}}(\mathrm{t})=V_{i n j}(\mathrm{t})\left(\frac{\mathrm{t}}{\tau_{o}}\right)\left\{1+\left[\frac{\sigma_{u p}(\mathrm{t})}{\varepsilon_{\mathrm{o}} \varepsilon_{\mathrm{r}}}\left(\frac{\mathrm{d}-\mathrm{R}\left(\mathrm{E}_{\mathrm{b}}\right)}{\mathrm{R}}\right)+\frac{\sigma_{\text {down }}(\mathrm{t})}{\varepsilon_{\mathrm{o}} \varepsilon_{\mathrm{r}}}\right] \mathrm{t}\right\}^{-1}$

$V_{s}(t)=V_{i n j}(\mathrm{t})\left(\frac{\mathrm{t}}{\tau_{o}}\right)\left\{1+\left[\frac{\sigma_{\text {down }}(\mathrm{t})}{\varepsilon_{\mathrm{o}} \varepsilon_{\mathrm{r}}}\right] \mathrm{t}\right\}^{-1}$

with the expression modified to include dissipation as

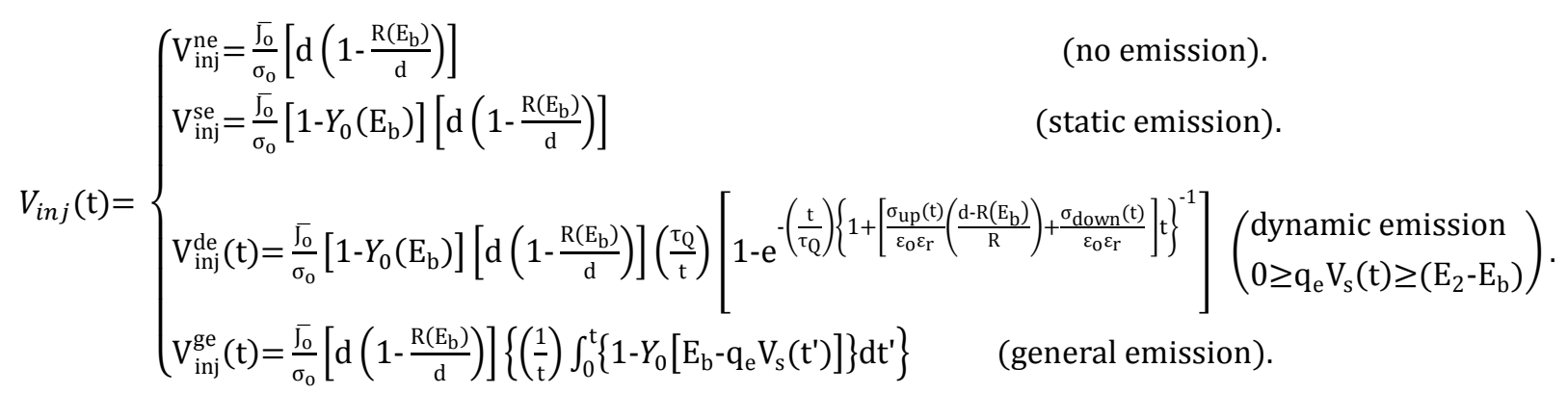

The above model for Fig. 3 makes direct ties to models based on double layers, with a time varying position for the embedded layer which is essentially the dynamic double layer model (DDLM) proposed by Melchinger and Hofmann $^{120}$, Cazaux $^{121}$ and Meyza ${ }^{122}$ [and employed and extended by Thomson ${ }^{47}$, Hoffmann ${ }^{25}$ and Wison ${ }^{123}$ of the USU MPG] to describe the charge dependence of electron emission in HDIM in terms of charge distributions in the region $0>\mathrm{Z}>\mathrm{R}$. The DDLM adds two additional charge layers: one to compensate for secondary electron emission at $\mathrm{Z}=0$, and another to account for secondary electrons reattracted to a positively charged dielectric of at a depth $\lambda_{S E} / 2$, where $\lambda_{S E}$ is equal to the average inelastic mean-free path of the secondary electrons. Refer to Ref 22 for further details.

\section{Conclusion and Future work}

The unified set of dynamic equations developed to describe the spatial and time-dependent voltage, electric fields, and currents can be applied to a broad range of electron transport experiments conducted in parallel plate geometry. This approach allows for the modeling of measurements for constant voltage (CVC), time-of-flight (TOF), radiation induced conductivity(RIC), surface voltage accumulation and decay (SVP); electrostatic discharge (ESD), and electron emission (SEE) for evolving electron transport, and related phenomena in HDIM over large ranges of time, electric field, temperature, absorbed dose, and adsorbed dose rate. A discussion of extensions to electroluminescence and AC conductivity have not been presented as the extension of the ideas presented here are beyond the scope of this paper. Key elements are the broad applications of the theoretical framework outlined in terms of injected charge and its effects on surface deposition, bulk deposition, and penetrating radiation for pulsed, stepped and periodic applied voltages/charge from either surface electrodes or electron beams. In particular the inclusion of dynamic charging, energy-dependent range theory, a comprehensive description of the DOS, and meanfield-trapped-controlled transport provide a simple and effective way to model the response of HDIM to conditions encountered in spacecraft operation.

This approach provides for the coupling of current USU MPG data, from several experimental systems, into a consistent complete picture of the physical processes. ${ }^{22-25,125}$, For each of the experimental systems there are simple analytical approximations to the transport equations that hold under specific circumstances. ${ }^{22-25}$ However, it has become clear that a consistent numerical implementation of the transport equations is required for a detailed universal description of observed behavior. It should be noted that a single theoretical model does describe many types of material response under many types of stimulus. A single numerical model can be constructed to predict material response simply by describing its physical parameters and environmental conditions. Major strides in this numerical effort have been made by Griseri ${ }^{49}$ and Laurant ${ }^{125}$ in conjunction with pulsed electro-acoustic (PEA) measurements.

Using a consisted theoretical picture, multiple materials, current data in the literature, and current data from the USU MPG across multiple experimental systems will provide a consistent study of HDIM their material parameters and spacecraft charging. 


\section{Acknowledgments}

We gratefully acknowledges the contributions to this body of work by the members of the Utah State University Materials Physics group over the last two decade, including Jerilyn Brunson, Justin Dekany, Jodie Corbridge Gillespie, Steven Hart, Ryan Hoffmann, Josh Hodges, Amberly Evans Jensen, Neal Nickles, Jennifer Albertsen Roth, Clint Thomson, and Greg Wilson who all made major contributions to this work.

\section{References}

1. Mandell, M., Katz, I., and Hilton, M. "NASCAP-2K Spacecraft Charging Models: Algorithms and Applications, in 2001: A Spacecraft Charging Odyssey," Proceed. of the 7th Spacecraft Charging Technology Conf., 23 “27 April, 2001, p. $499-507$. 1996.

2. Hastings, D., and Garrett, H. Spacecraft-Environment Interactions: Cambridge Atmospheric and Space Science Series,

3. Novikov, L. S., Mileev, V. N., Voronina, E. N., Galanina, L. I., Makletsov, A. A., and Sinolits, V. V. "Radiation effects on spacecraft materials," Poverkhnost, No. 3, 2009, pp. 32-48.

4. Griseri, V., Perrin, C., Fukunaga, K., Maeno, T., Payan, D., Levy, L., and Laurent, C. "Analysis of electron behavior in polymeric films during electronic irradiation," Electrical Insulation and Dielectric Phenomena, 2005. CEIDP'05. 2005 Annual Report Conference on, 2005, pp. 645-648.

5. Dennison, J. R., Brunson, J., Swaminathan, P., Green, N. W., and Frederickson, A. R. "Methods for high resistivity measurements related to spacecraft charging," IEEE Transactions On Plasma Science Vol. 34, No. 5, Pt. 2, 2006 , pp. $2191-2203$.

6. Garrett, H. B., I. Jun, A. A. Shapiro,. "Interstellar Space Missions: Ultra-Reliability Requirements and Engineering Issues," 45th AIAA Aerospace Sciences Meeting and Exhibit. Reno, Nevada, 2007.

7. Dennison, J.R. "Interaction of the Space Environment with Spacecraft Materials," Invited Seminar. Alcatel Space Facility, Cannes, France, 2004.

8. Dennison, J.R., Chang, W.-Y., Nickles, N., Kite, J., Thomson, C. D., Corbridge, J., and Ellsworth, C. " Electronic Properties of Materials with Application to Spacecraft Charging." Published by NASA electronically at URL: http://see.msfc.nasa.gov/scck/ 2002, p. the work is comprised of 16 individual Materials Reports with a combined length of 672 pages.

9. Dennison, J.R., Chang, W.-Y., Nickles, N., Kite, J., and Thomson, C. D. "'Electronic Properties of Materials with Application to Spacecraft Charging"," Final Report Part 1: Instrumentation; Methods and Analysis NASA.

10. Davies, R., and Dennison, J.R. "Evolution of Secondary Electron Emission Characteristics of Spacecraft Surfaces," Journal of Spacecraft and Rockets Vol. 34, 1997, pp. 571-573.

11. Dennison, J.R., Thomson, C., and Sim, A. "The effect of low energy electron and UV/VIS radiation aging on the electron emission properties and breakdown of thin-film dielectrics," Proceedings of the 8th 2004 IEEE Dielectrics and Electrical Insulation Society (DEIS) International Conference on Solid Dielectrics (ICSD). Vol. 2, Piscataway, NJ, 2004, pp. 967-971.

12. Sim, A. Dennison, J.R., and Thomson, C. "Evolution of the Electron Yield Curves of Insulators as a Function of Impinging Electron Fluence and Energy," Proceedings of the 9th Spacecraft Charging Technology Conference. Epochal Tsukuba, Japan, 2005, p. 19.

13. Abbott, J., and Dennison, J.R. "Methods for Determining Crossover Energies in Insulating Materials," Utah State University Student Showcase. Logan, UT, 2005.

14. Kite, J., Dennison, J. R., "Change, W. Y., and Davies, R. E. "Effects of Evolving Surface Contamination On Spacecraft Charging," American Physical Society Four Corner Sectional Meeting. Fort Collins, CO, 2000.

15. Dennison, J.R., Sim, A., Brunson, J., Steven Hart, Jodie Gillespie, Justin Dekany, Charles Sim and Dan Arnfield, "Engineering Tool for Temperature, Electric Field and Dose Rate Dependence of High Resistivity Spacecraft Materials Paper Number," AIAA-2009-0562, Proceedings of the 47th American Institute of Aeronautics and Astronautics Meeting on Aerospace Sciences, 2009.

16. Dennison, J.R., J. B., Prasanna Swaminathan, Nelson Green, and Frederickson, A. R. "Methods for Resistivity Measurements Related to Spacecraft Charging," IEEE Transaction on Plasma Science Vol. 34, No. 5, 2006, pp. $2191-2203$.

17. Brunson, J. "Measurement of Charge Decay Time and Resistivity of Spacecraft Insulators Using Charge Storage Method and Application to Theoretical Modeling of Charging Behavior of Insulators," Physics. Vol. PhD, Utah State University, Logan, UT, 2009, p. 215.

18. Hoffmann, R., Dennison, J.R., Albretsen, J., "Flux and Fluence Dependence of Electron Emission for High-yield, Highresistivity Materials: Implications for Spacecraft Charging," Proceedings of the 47th American Institute of Aeronautics and Astronomics Meeting on Aerospace Sciences. 2009, pp. AIAA-2009-0348.

19. Swaminathan, P., "Measurement of Charge Storage Decay Time and Resistivty of Spacecraft Insulators," Electrical Engineering. Vol. MS, 2004.

20. Fredrickson, A., and Dennison, J.R. "Measurement of conductivity and charge storage in insulators related to spacecraft charging," IEEE Transactions on Nuclear Science Vol. 50, No. 6, 2003, pp. 2284-2291.

21. Dennison, J.R., Jodie Gillespie, Joshua Hodges, RC Hoffmann, J Abbott, Alan W. Hunt and Randy Spalding, "Radiation Induced Conductivity of Highly-Insulating Spacecraft Materials," in Application of Accelerators in Research and Industry, American Institute of Physics 
Conference Proceedings Series, Vol. 1099, ed. Floyd D. McDaniel and Barney L. Doyle,(American Institute of Physics, Melveille, NY, 2009), pp. 203-208.

22. Sim, A., "A unified model of Charge Transport in Insulating Materials." Ph.D Dissertation, Utah State University, Physics Dept, Logan, UT, 2013.

23. Gillespie, J., "Measurements of the Temperature Dependence of Radiation Induced Conductivity in Polymeric Dielectrics", M.S. Thesis, Utah State University, Physics Dept, Logan, UT, 2013.

24. Hodges, J. L. "In Situ Measurements of Electron Beam Induced Surface Voltage of Highly Resistive Materials," Physics.

M.S., Utah State University, Logan, 2012

25. Hoffmann, R. "Electron-Induced Electron Yields of Uncharged Insulating Materials" Physics. Vol. M.S., Utah State University Logan, UT 2010, p. 183.

26 Dennison, J.R., Gillespie, J., Hodges, J., Hoffmann, R. C., Abbott, J., Hart, S., and Hunt, A. W. "Temperature Dependence of Radiation Induced Conductivity in Insulators," Submitted IEEE Transactions on Plasma Science Vol. NA, No. NA, 2008.

27. Wintle, H. J. "Charge Motion in Technical Insulators: Facts, Fancies and Simulations," IEEE Transactions on Dielectrics \& Electrical Insulation Vol. 10, No. 5, 2003, p. 16p.

28. Anderson, P. W. "Absence of diffusion in certain random lattices," Physical Review Vol. 109, 1958, pp. 1492-1505.

29. Dennison, J. R., and Brunson, J. "Temperature and electric field dependence of conduction in low-density polyethylene,"

IEEE Transactions on Plasma SCIENCE Vol. 36, No. 5, Pt. 2, 2008, pp. 2246-2252.

30. H. Bässler. "Charge Transport in Disordered Organic Photoconductors a Monte Carlo Simulation Study," physica status solidi (b) Vol. 175, No. 1, 1993, pp. 15-56.

31. Mott, N. F. "Transport in Disordered Materials," Physical Review Letters Vol. 31, No. 7, 1973, p. 466.

32. Montanari, G. C., Mazzanti, G., Palmieri, F., Motori, A., Perego, G., and Serra, S. "Space-charge trapping and conduction in LDPE, HDPE and XLPE," Journal of Physics D Applied Physics Vol. 34, No. 18, 2001, pp. $2902-2911$.

33. Rose, A. "Space-Charge-Limited Currents in Solids," Physical Review Vol. 97, No. 6, 1955, p. 1538.

34. Schmidlin, F. W. "Kinetic theory of hopping transport. I. The formalism and its relationship to random walks," Philosophical Magazine B Physics of Condensed Matter Statistical Mechanics, Electronic, Optical and Magnetic Properties Vol. 41, No. 5, 1980, pp. 535-70.

35. Wintle, H. J. "Charge motion and trapping in insulators: surface and bulk effects," Electrical Insulation, 1998.

Conference Record of the 1998 IEEE International Symposium on. Vol. 1, 1998, pp. 1-11 vol.1.

36. Böttger, H., and Bryksin, V. V. Hopping conduction in solids. Deerfield Beach, FL: VCH, 1985.

37. Apsley, N., and Hughes, H. P. "Temperature and field-dependence of hopping conduction in disordered systems," Philosophical Magazine Vol. 30, No. 5, 1974, pp. 963-72.

38. Wintle, H. J. "Basic physics of insulators," IEEE Trans. Electr. Insul. Vol. 25, No. 1, 1990, pp. 27-44.

39. Ambegaokar, V., Halperin, B. I., and Langer, J. S. "Hopping Conductivity in Disordered Systems," Physical Review B

Vol. 4, No. 8, 1971, p. 2612.

40. Miller, A., and Abrahams, E. "Impurity conduction at low concentrations," Phys. Rev. Vol. 120, 1960, pp. 745-55.

41. Baranovskii, S., and Rubel, O. "Charge transport in disordered materials," Springer Handb. Electron. Photonic Mater., 2006, pp. 161-186.

42. Schmidlin, F. W. "Theory of trap-controlled transient photoconduction," Physical Review B Solid State Vol. 16, No. 6, 1977, pp. 2362-85.

43. Cornet, N., Goeuriot, D., Guerret-Piecourt, C., Juve, D., Treheux, D., Touzin, M., and Fitting, H.-J. "Electron beam charging of insulators with surface layer and leakage currents," Journal of Applied Physics Vol. 103, No. 6, 2008 , p. 064110.

44. Wilson, G., and Dennison, J. R. "Approximation of Range in Materials as a Function of Incident Electron Energy," Plasma Science, IEEE Transactions on Vol. 40, No. 2, 2012, pp. 305-310.

45. Fitting, H. J., Glaefeke, H., and Wild, W. "Creation energies for secondary electrons," Kristall und Technik Vol. 14, No. 3, 1979, pp. K13-K17.

46. Davies, R. E., and Dennison, J. R. "Effects of the Evolution of Spacecraft Surfaces on Secondary Electron Emission and Spacecraft Charging," 6th Spacecraft Charging Technology Conference. Air Force Research Laboratory Science Center, Hanscom Air Force Base, MA, 1998.

47. Thomson, C. "Measurements of the Secondary Electron Emission Properties of Insulators," Physics. Vol. PhD, Utah State University, Logan, UT, 2004.

48. Clerc, S., Dennison, J. R., Hoffmann, R., and Abbott, J. "On the Computation of Secondary Electron Emission Models," IEEE Transactions on Plasma Science Vol. 34, 2006, p. 7p.

49. Dennison, J. R., Brunson, J., Swaminathan, P., Green, N. W., and Robb Frederickson, A. "Methods for High Resistivity Measurements Related to Spacecraft Charging," IEEE Transactions on Plasma Science Vol. 34, 2006, p 13.

50. Kite, J. "Secondary Electron Production and Transport Mechanisms by Measurement of Angle-Energy Resolved Cross Sections of Secondary and Backscattered Electron Emission," Physics. Vol. PhD, Utah State University, Logan, UT, 2007.

51. Arkhipov, V. "Radiation-induced conductivity and charge storage in irradiated dielectrics," Journal of Applied Physics Vol. 26, 1993, pp. 1298-1300.

52. Sessler, G. Electrets: Springer-Verlag, 1987

53. Gross, B. Charge Storage in Solid Dielectrics, 1964.

54. Gross, B. "Charge storage and transport in solid dielectrics. (The case of irradiated polymers)," Annual Report Conference on Electrical Insulation and Dielectric Phenomena, 1978, pp. 55-70. 
55. Gross, B. "Radiation-induced charge storage and polarization effects [in dielectrics]," Topics in Applied Physics Vol. 33, No. Electrets, 1980, pp. 217-84.

56. Gross, B., and Nablo, S. V. "High potentials in electron-irradiated dielectrics," Journal of Applied Physics Vol. 38, No. 5, 1967, pp. 2272-5.

57. Gross, B., and Nunes de Oliveira, L. "Transport of excess charge in electron-irradiated dielectrics," Journal of Applied Physics Vol. 45, No. 11, 1974, pp. 4724-9.

58. Gross, B., Giacometti, J. A., and Ferreira, G. F. L. "Charge storage and transport in electron-irradiated and coronacharged dielectrics," IEEE Transactions on Nuclear Science Vol. NS28, No. 6, 1981, pp. 4513-22.

59. Gross, B., Faria, R. M., and Ferreira, G. F. L. "Radiation-induced conductivity of Teflon irradiated by x rays," Journal of Applied Physics Vol. 52, No. 2, 1981, pp. 571-7.

60. Gross, B., and Perlman, M. "Short-Circuit Currents in Charged Dielectrics and Motion of Zero-Field Planes," Journal of Applied Physics Vol. 43, 2003, p. 853.

61. Giacometti, J. A., Ferreira, G. F. L., and Gross, B. "Negative charge transport in fluoroethylenepropylene by the constant current method," Physica Status Solidi A: Applied Research Vol. 88, No. 1, 1985, pp. 297-307.

62. Gross, B., and de Oliveira, L. "Transport of excess charge in electron-irradiated dielectrics," Journal of Applied Physics Vol. 45, 2003, p. 4724.

63. Kastner, M. A., and Monroe, D. "The relationship between transient and steady-state photoconductivity in amorphous semiconductors," Sol. Energy Mater. Vol. 8, No. 1-3, 1982, pp. 41-52.

64. Pai, D., and Springett, B. "Physics of electrophotography," Reviews of Modern Physics Vol. 65, No. 1, 1993, pp. 163211.

65. Rose, A. "An Outline of Some Photoconductive Processes," RCA Review Vol. 12, 1951, pp. 362-414.

18. Fowler, J. "X-Ray Induced Conductivity in Insulating Materials," Proceedings of the Royal Society of London. Series A, Mathematical and Physical Sciences (1934-1990) Vol. 236, No. 1207, 1956, pp. 464-480.

66. Arkhipov, V. I., Vannikov, A. V., Mingaleev, G. S., Popova, Y. A., Rudenko, A. I., Saenko, V. S., and Tyutnev, A. P. "Transient Photocurrent Due to Step-Function Excitation in Disordered Materials - Computer-Simulation and Analytical Treatment," Journal of Physics D-Applied Physics Vol. 17, No. 7, 1984, pp. 1469-1475.

67. Arkhipov, V. I. "Kinetics of pair recombination in amorphous materials," Fizika i Tekhnika Poluprovodnikov (SanktPeterburg) Vol. 20, No. 3, 1986, pp. 556-8.

68. Tiedje, T. "A physical interpretation of dispersive transport in disordered semiconductors," Solid State Communications Vol. 37, 1980, pp. 49-52.

69. Mott, N. F. "Electronic processes in glasses," 1977, pp. 101-7.

70. Rudenko, A. I., and Arkhipov, V. I. "Drift and diffusion in materials with traps. I. Quasiequilibrium transport regime," Philosophical Magazine B Physics of Condensed Matter Statistical Mechanics, Electronic, Optical and Magnetic Properties Vol. 45, No. 2, 1982, pp. 177-87.

71. Arkhipov, V. I., Gromov, V. V., Mamonov, M. N., Rozno, A. G., and Rudenko, A. I. "Gamma-ray electrification of dielectrics," Atomnaya Energiya Vol. 62, No. 2, 1987, pp. 134-6.

72. Arkhipov, V. I., Nikitenko, V. R., and Rudenko, A. I. "Nonequilibrium photodielectric effect in disordered materials," Fizika i Tekhnika Poluprovodnikov (Sankt-Peterburg) Vol. 22, No. 3, 1988, pp. 544-7.

73. Arkhipov, V. I., and Nikitenko, V. R. "Langevin-recombination-controlled explosive kinetics of electroluminescence in organic semiconductors," Semiconductors (Translation of Fizika i Tekhnika Poluprovodnikov (Sankt-Peterburg)) Vol. 33, No. 8, 1999, pp. 862-864.

74. Weaver, L., Shultis, J., and Faw, R. "Analytic solutions of a model for radiation-induced conductivity in insulators," Journal of Applied Physics Vol. 48, 1977, p. 2762.

75. Tyutnev, A. P., Vannikov, A. V., Saenko, V. S., Likhovidov, V. S., and Pozhidaev, E. D. "Electrical-Conductivity of Films of Polyvinylcarbazole Induced by Electron-Bombardment," High Energy Chemistry Vol. 16, No. 6, 1982, pp. 386-390.

76. Tyutnev, A. P., Saenko, V. S., Vannikov, A. V., and Mingaleev, G. S. "Radiation-Induced Conductivity in Polyethylene," Physica Status Solidi a-Applied Research Vol. 78, No. 2, 1983, pp. 689-696.

77. Tyutnev, A. P., Saenko, V. S., Valetskii, P. M., Kim, V. A., Safonov, G. P., Pozhidaev, Y. D., Vinogradova, S. V., and Korshak, V. V. "Electrical Phenomena under the Effect of Low-Energy Electrons on Polyarylates," Vysokomolekulyarnye Soedineniya Seriya A Vol. 25, No. 4, 1983, pp. 856-861.

78. Tyutnev, A. P., Saenko, V. S., Dunaev, A. F., Pozhidaev, E. D., and Vannikov, A. V. "Temperature-Dependence of Transient Radiation-Induced Conductivity in Polymers," Physica Status Solidi a-Applied Research Vol. 85, No. 2, 1984, pp. 591602.

79. Tyutnev, A. P., Abramov, V. N., Saenko, V. S., Dubenskov, P. I., Vannikov, A. V., and Pozhidaev, E. D. "RadiationInduced Conductivity in Foamed Dielectrics," Physica Status Solidi a-Applied Research Vol. 88, No. 2, 1985, pp. 673-680.

80. Tyutnev, A. P., Vannikov, A. V., and Saenko, V. S. "Radiation-Dielectric Effect in Polymers," Vysokomolekulyarnye Soedineniya Seriya B Vol. 27, No. 2, 1985, pp. 98-103.

81. Tyutnev, A. P., Ikhsanov, R. S., Saenko, V. S., and Pozhidaev, E. D. "A theoretical description of geminal recombination and radiation-pulse-induced electric conduction in polymers in the approximation of probability of survival of ionic pairs," Russian Journal of Physical Chemistry B Vol. 1, No. 6, 2007, pp. 661-669.

82. Dunaev, A. F., Tyutnev, A. P., Saenko, V. S., Makeev, S. N., Filatov, N. I., and Pozhidaev, E. D. "Pulsed Reactor Induced Conductivity in Polymers," Physica Status Solidi a-Applied Research Vol. 130, No. 2, 1992, pp. 391-396. 
83. Arkhipov, V. I., Nikitenko, V. R., and Tyutnev, A. P. "Radiation-induced dielectric effect in polymers," Chemical Physics Reports Vol. 16, No. 2, 1997, pp. 301-309.

84. Dissado, L. A., and Fothergill, J. C. Electrical degradation and breakdown in polymers. London: P. Peregrinus, 1992.

85. Dissado, L. A., Griseri, V., Peasgood, W., Cooper, E. S., Fukunaga, K., and Fothergill, J. C. "Decay of space charge in a glassy epoxy resin following voltage removal," IEEE Trans. Dielectr. Electr. Insul. Vol. 13, No. 4, 2006, pp. 903-916.

86. Wilcox, P. "A Dielectric Loss Model Based on Interfacial Electron Tunneling," Canadian Journal of Physics Vol. 50, 1971.

87. Liufu, D., Wang, X. S., Tu, D. M., and Kao, K. C. "High-field induced electrical aging in polypropylene films," Journal of Applied Physics Vol. 83, No. 4, 1998, pp. 2209-2214.

88. Kao, K. C., ed. Dielectric Phenomena in Soilds: Elsevier Academic Press, 2004.

89. Toomer, R., and Lewis, T. J. "Charge trapping in corona-charge polyethylene films," Journal of Physics D: Applied Physics Vol. 13, No. 7, 1980, p. 1343.

90. Wintle, H. J. "Absorption currents and steady currents in polymer dielectrics," J. Non-Cryst. Solids Vol. 15, No. 3, 1974, pp. 471-486.

91. Wintle, H. J. "Time dependent photocurrents in polyethylene," J. Polym. Sci., Polym. Phys. Ed. Vol. 12, No. 10, 1974, pp. 2135-51.

92. Walden, R. H. "A Method for the Determination of High-Field Conduction Laws in Insulating Films in the Presence of Charge Trapping," J. Appl. Phys Vol. 43, No. 3, 1971.

93. Fothergill, J. C., ed. Space Charge in Solid Dielectrics: The Dielectrics Society, 1998.

94. Touzin, M. "Electron beam charging of insulators: A self-consistent flight-drift model," Journal of Applied Physics Vol. 99, 2006.

95. Fitting, H. J. "Time-dependent start-up and decay of secondary electron emission in dielectrics," Journal of Applied Physis Vol. 108, 2010.

96. Avini, E. "Trap generation and occupation in stressed gate oxides under spatially variable oxide electric field," Applied Physics Letters Vol. 51, No. 22, 1987.

97. DiMaria, D. J. "Capture and emission of electrons at 2.4-eV-deep trap level in SiO2 films," Physical Review B Vol. 11, No. $12,1975$.

98. Nissan-Cohen, Y. "Trap generation and occupation dynamics in Si02 under charge injection stress," J. Appl. Phys Vol. 60, No. 6, 1986.

99. Orenstein, J., and Kastner, M. A. "Thermalization and recombination in amorphous semiconductors," Solid State Commun. Vol. 40, No. 1, 1981, pp. 85-9.

100. Reimer, L. "Scanning electron microscopy: physics of image formation and microanalysis," Measurement Science and Technology Vol. 11, 2000, p. 1826.

101. Spencer, L. "Theory of electron penetration," Physical Review Vol. 98, No. 6, 1955, pp. 1597-1615.

102. Alig, R., and Bloom, S. "Electron-hole-pair creation energies in semiconductors," Physical Review Letters Vol. 35, No. 22, 1975, pp. 1522-1525.

103. Pines, D. Elementary excitations in solids: lectures on protons, electrons, and plasmons: Westview Press, 1999.

104. Tanuma, S., Powell, C. J., and Penn, D. R. "Calculations of Electron Inelastic Mean Free Paths (IMFP's) VI. Analysis of the Gries Inelastic Scattering Model and Predictive IMFP Equation," Sur. Inter. Anal. Vol. 25, No. 25, 1997.

105. Bethe, H., and Heitler, W. "On the Stopping of Fast Particles and on the Creation of Positive Electrons," Proceedings of the Royal Society of London. Series A, Containing Papers of a Mathematical and Physical Character Vol. 146, No. 856, 1934, pp. 83-112. 1989.

106. DC, Joy., and S., Luo. "An empirical stopping power expression for low energy electrons," Scanning Vol. 11, No. 176,

107. Tanuma, S., Powell, C., and Penn, D. "Calculations of stopping powers of $100 \mathrm{eV}$ to $30 \mathrm{keV}$ electrons in 10 elemental solids," Surface and Interface Analysis Vol. 37, No. 11, 2005, pp. 978-988.

108. National Institute of Standards and Technology, 2010, "ESTAR, Stopping Power and Range Tables for Electrons," (http://physics.nist.gov/PhysRefData/Star/Text/ESTAR.html).

109. Ziegler, J.F., "RBS/ERD simulation problems: Stopping powers, nuclear reactions and detector resolution," Nuclear Instruments and Methods B 132 (1997) 377-390.

110. Rodgers, D. J.; Ryden, K. A.; Wrenn, G. L.; Lévy, L.; Sørensen, J. "Fitting of material parameters for DICTAT internal dielectric charging simulations using DICFIT," Proc. 9th Intern. Symp. on Materials in a Space Environment, 16-20 June 2003, Noordwijk, The Netherlands. Compiled by K. Fletcher. ESA SP-540, Noordwijk, Netherlands: ESA Publications Division,. 2003, p. $609-613$.

111. Kim, W. "NUMIT 2.0: The Official Release of the JPL's Internal Charging Code," Proceedings of the 12th Spacecraft Charging Technology Conference, (Kitakyushu, Japan, May 14-18, 2012).

112. Beecken, B.P. and B.M. Wallin, "Modeling of Deep-dielectric Spacecraft Charging in Realistic Environments with NUMIT2," Paper AIAA-2011, Proc. of 49th AIAA Meeting on Aerospace Sci., Orlando, FL, 2011.

113. National Institute of Standards and Technology, 2010, "NIST Electron Inelastic-Mean-Free-Path Database: Version 1.1," (http://www.nist.gov/data/nist71.htm).

114. Wilson, G., "Models and Measurements of Electron Ranges at Low and Intermediate Energies," MS Thesis, Utah State Univ., Logan, UT, August 2013. 
115. Evans, R. D. "The Atomic Nucleus," Internat. Ser. In Pure and Appl. Phys., McGraw-Hill, New York, 1955.

116. Pines, D., Elementary Excitations in Solids, Addison-Wesley, Reading, MA. , 1963

117. Zangwill, A. Physics at surfaces. Cambridge, MA: Cambridge Univ Pr, 1988.

118. Somorjai, G. Chemistry in Two Dimensions. Ithica: Cornell Press, 1981.

119. Penn, D. "Electron mean free paths for free-electron-like materials," Physical Review B Vol. 13, No. 12, 1976, pp. 5248-5254

120. Melchinger, A., and Hofmann, S., "Dynamic Double Layer Model: Description of Time Dependent Charging Phenomena in Insulators under Electron Beam Irradiation," J. Appl. Phys. 78, 6224, 1995.

121. Cazaux, J. "Some considerations on the secondary electron emission, d, from e irradiated insulators," Journal of Applied Physics Vol. 85, 1999, p. 1137.

122. Meyza, X., Goeuriot, C., Guerret-Piecourt, D., Treheux, and H. Fitting, "Secondary Electron Emission and SelfConsistent Charge Transport and Storage in Bulk Insulators: Application to Alumina," J. Appl. Phys. 94, $5384,2003$.

123. Wilson, G., Evans, A., Dekany, J., "Charging Effects of Multilayered Dielectric Spacecraft Materials: Surface Voltage, Discharge and Arcing," IEEE Trans. on Plasma Sci., 2013, in press.

124. Justin Dekany, Alec M. Sim, Jerilyn Brunson, and JR Dennison, "Electron Transport Models and Precision Measurements in a Constant Voltage Chamber," IEEE Trans. on Plasma Sci., 2013, in press.

125. Laurent, C. "Charge dynamics in polymeric materials and its relation to electrical ageing," Electrical Insulation and Dielectric Phenomena (CEIDP), 2012 Annual Report Conference on. pp. 1-20. 